\title{
THE PLANCHEREL FORMULA FOR COMPLEX SEMISIMPLE LIE GROUPS
}

\author{
BY \\ HARISH-CHANDRA
}

1. Introduction. Let $G$ be a connected semisimple Lie group and $\pi$ an irreducible unitary representation of $G$ on a Hilbert space. Let $C_{c}^{\infty}(G)$ denote the class of all (complex-valued) functions on $G$ which vanish outside a compact set and which are indefinitely differentiable everywhere. Then we have seen in [8] that for any $f \in C_{c}^{\infty}(G)$ the operator

$$
\int f(x) \pi(x) d x
$$

( $d x$ is the Haar measure on $G$ ) has a trace which we shall denote by $T_{\pi}(f)$. The mapping $T_{\pi}: f \rightarrow T_{\pi}(f)$ is then a distribution which depends only on the equivalence class of $\pi$. Hence if $\mathcal{E}$ is the set of all equivalence classes of irreducible unitary representations of $G$, we have a distribution $T_{\omega}$ defined for each $\omega \in \mathcal{E}$. Our object is to find a (positive) measure $d \omega$ on $\mathcal{E}$ such that

$$
f(1)=\int_{\varepsilon} T_{\omega}(f) d \omega \quad\left(f \in C_{c}^{\infty}(G)\right)
$$

at least in case $G$ is a complex group. Let $f^{\prime}$ be the function $\left.{ }^{1}\right) x \rightarrow \operatorname{conj}\left(f\left(x^{-1}\right)\right)$ $(x \in G)$ and let $F=f^{\prime} * f$ where * denotes group convolution. Then

$$
F(x)=\int f^{\prime}(y) f\left(y^{-1} x\right) d y=\int \operatorname{conj}(f(y)) f(y x) d y
$$

and therefore $\int|f(x)|^{2} d x=F(1)=\int \varepsilon T_{\omega}\left(f^{\prime} * f\right) d \omega$. But

$$
T_{\pi}\left(f^{\prime} * f\right)=\left\|\int f(x) \pi(x) d x\right\|^{2} \quad(\pi \in \omega)
$$

where $\|\cdot\|$ denotes the Hilbert-Schmidt norm. Hence

$$
\int|f(x)|^{2} d x=\int \varepsilon^{N_{\omega}(f) d \omega}
$$

where $N_{\omega}(f)=\left\|\int f(x) \pi(x) d x\right\|^{2}$ for any $\pi \in \omega$. This formula may be regarded as the analogue of the Plancherel formula for abelian groups or of the PeterWeyl completeness relation for compact groups (see Gelfand and Naimark $[3$, p. 198]).

Received by the editors May 11, 1953.

(1) For any complex number $c$ we denote the conjugate of $c$ by conj $c$. 
Although the final formula of this paper is applicable only when $G$ is complex, the complex structure of $G$ plays no essential role in the earlier stages of the computation. Hence, in the hope that the present method could perhaps be extended to arbitrary semisimple Lie groups, we shall avoid making the assumption about the complexity of $G$ until it becomes absolutely necessary.

2. Some preliminary results. Let $\mathrm{g}_{0}$ be the Lie algebra of $G$ over the field $R$ of real numbers. We define $\mathfrak{f}_{0}, \mathfrak{h}_{\mathfrak{p}_{0}}$, and $\mathfrak{n}_{0}$ as in $[6, \S 2]$. Let $K, A_{+}, N$ be the analytic subgroups of $G$ corresponding to $\mathfrak{f}_{0}, \mathfrak{h}_{\mathfrak{p}_{0}}$, and $\mathfrak{n}_{0}$ respectively. Then $K$ is closed and it contains the center $Z$ of $G$. Let $\mathfrak{f}_{0}^{\prime}=\left[\mathfrak{t}_{0}, \mathfrak{t}_{0}\right]$ be the derived algebra and $\mathfrak{c}_{0}$ the center of $\mathfrak{f}_{0}$. We denote by $K^{\prime}$ and $D$ the analytic subgroups of $K$ corresponding to $f_{0}^{\prime}$ and $\mathfrak{c}_{0}$ respectively. $K^{\prime}$ is semisimple and compact and $D$, being the connected component of the centralizer of $K^{\prime}$ in $K$, is closed. Put $G^{*}=G / D \cap Z$ and let $x \rightarrow x^{*}$ denote the natural mapping of $G$ on $G^{*}$. Then $K^{*}$ is compact. We shall say that a representation $\pi$ of $G$ on a Banach space is permissible if $\pi(z)$ is a scalar multiple of the unit operator for all $z \in Z \cap D$.

Let $\Omega$ be the set of all equivalence classes of finite-dimensional simple representations of $K$.

Lemma 1. Let $\pi$ be a representation of $G$ on a Hilbert space $\mathfrak{S}$. For any $\mathfrak{D} \in \Omega$ let $\mathfrak{S}_{\mathfrak{D}}$ denote the subspace consisting of all those elements in $\mathfrak{S}$ which transform under $\pi(K)$ according to $\mathfrak{D}$. Suppose the following two conditions are fulfilled.

(i) $\pi$ is permissible.

(ii) There exists an integer $N$ such that $\operatorname{dim} \mathfrak{S}_{\mathfrak{D}} \leqq N d(\mathfrak{D})^{2}$ for all $\mathfrak{D} \in \Omega$. (Here $d(\mathfrak{D})$ is the degree of any representation in $\mathfrak{D}$.)

Then if $f \in C_{c}^{\infty}(G)$ the operator $\int f(x) \pi(x) d x$ fulfills the conditions of Lemma 1 of $[8]$.

The proof is exactly the same as that given in $\$ 5$ of [8]. We can therefore conclude from Lemma 1 of [8] that $\int f(x) \pi(x) d x$ has a trace. We denote this trace by $T_{\pi}(f)$ and prove exactly as in $\S 5$ of [8] that the mapping $T_{\pi}: f \rightarrow T_{\pi}(f)$ $\left(f \in C_{c}^{\infty}(G)\right)$ is a distribution which depends only on the equivalence class of $\pi$. We shall call $T_{\pi}$ the character of $\pi$.

LeMma 2. Let $\pi$ be a permissible unitary representation of $G$ on a Hilbert space $\mathfrak{S}$. Suppose $\operatorname{dim} \mathfrak{S}_{\mathfrak{D}}<\infty$ for every $\mathfrak{D} \in \Omega$. Then $\mathfrak{T}$ can be written as a sum $(2)$ of a countable number of mutually orthogonal closed subspaces each of which is invariant and irreducible under $\pi(G)$.

By going over to the simply connected covering group of $G$ it follows that

$\left.{ }^{2}\right)$ The sum here is understood in the sense of Hilbert space theory. It denotes the closure of the algebraic sum. 
for any homomorphism $\xi$ of $Z$ into the field $C$ of complex numbers we can find a homomorphism $\eta$ of $K$ into $C$ such that $\eta(z)=\xi(z)$ for $z \in D \cap Z$ (see $\S 9$ of [6]). Therefore in particular we can choose $\eta$ such that $\pi(z)=\eta(z) \pi(1)$ $(z \in D \cap Z)$. Then $\eta\left(u^{-1}\right) \pi(u)(u \in K)$ depends only on $u^{*}$ and if we denote it by $\pi^{*}\left(u^{*}\right)$ the mapping $\pi^{*}: u^{*} \rightarrow \pi^{*}\left(u^{*}\right)$ is a representation of $K^{*}$ on $\mathfrak{S}$. Let $\Omega^{*}$ be the set of all equivalence classes of finite-dimensional simple representations of $K^{*}$. We denote by $\mathfrak{S}_{\mathfrak{D}}^{*}\left(\mathfrak{D} \in \Omega^{*}\right)$ the subspace of those elements in $\mathfrak{S}$ which transform under $\pi^{*}\left(K^{*}\right)$ according to $\mathfrak{D}$. Then it is clear that $\operatorname{dim} \mathfrak{S}_{\mathfrak{D}}^{*}<\infty$. Since $K^{*}$ is a compact Lie group, $\Omega^{*}$ is a countable set. Hence we can arrange its elements in a sequence $\mathfrak{D}_{i}(i \geqq 1)$. We shall now define a sequence of closed subspaces $\mathfrak{W}_{j}(j \geqq 0)$ with the following properties:

(i) $\mathfrak{S}_{j}$ is invariant under $\pi(G)$.

(ii) $\mathfrak{S}_{j} \supset \sum_{i=1}^{j} \mathfrak{S}_{\mathfrak{D}_{i}}^{*}$.

(iii) $\mathfrak{S}_{j+1} \supset \mathfrak{S}_{j}$ and the orthogonal complement of $\mathfrak{S}_{j}$ in $\mathfrak{S}_{j+1}$ is the sum of a finite number of mutually orthogonal closed spaces each of which is invariant and irreducible under $\pi(G)$.

We proceed by induction on $j$. Put $\mathfrak{S}_{0}=\{0\}$. Now suppose $\mathfrak{S}_{j}$ has been defined. Let $V_{j}$ be the orthogonal complement of $\mathfrak{S}_{j}$ in $\mathfrak{S}$. Since $\pi$ is unitary, $V_{j}$ is invariant under $\pi(G)$. Let $V_{j, \mathscr{D}}=V_{j} \cap \mathfrak{S}_{\mathfrak{D}}^{*}\left(\mathfrak{D} \in \Omega^{*}\right)$. It is clear that $\mathfrak{S}_{\mathfrak{D}}^{*}=V_{j, \mathfrak{D}}+\mathfrak{S}_{j} \cap \mathfrak{S}_{\mathfrak{D}}^{*}\left(\mathfrak{D} \in \Omega^{*}\right)$. If $V_{j, \mathfrak{D}_{j+1}}=\{0\}$ we put $\mathfrak{S}_{j+1}=\mathfrak{S}_{j}$ and all the three conditions are verified. Now suppose $V_{j, \mathscr{D}_{j+1}} \neq\{0\}$. Then

$$
0<\operatorname{dim} V_{j, \mathscr{D}_{j+1}} \leqq \operatorname{dim} \mathfrak{S}_{\mathfrak{D}_{j+1}}^{*}<\infty .
$$

We shall now define a sequence of mutually orthogonal closed subspaces $W_{r}$ $(r \geqq 0)$ which are invariant and irreducible under $\pi(G)$. Put $W_{0}=\{0\}$ and suppose $W_{i}(0 \leqq i \leqq r)$ have already been defined. Let $U_{r}$ be the orthogonal complement of $W_{1}+\cdots+W_{r}$ in $V_{j}$. If $U_{r} \cap \mathfrak{S}_{\mathfrak{D}_{j+1}^{*}}^{*}=\{0\}$ put $W_{r+1}=\{0\}$. So now let us suppose $\operatorname{dim}\left(U_{r} \cap \mathfrak{S}_{\mathfrak{D}_{j+1}^{*}}^{*}\right)>0$. Let $\Sigma$ be the collection of all closed subspaces $U$ of $U_{r}$ which are invariant under $\pi(G)$ and such that $U \cap \mathfrak{S}_{\mathfrak{D}_{j+1}}^{*} \neq\{0\}$. Choose $U \in \Sigma$ such that $s=\operatorname{dim} U \cap \mathfrak{S}_{\mathfrak{D}_{j+1}^{*}}^{*}$ has the least possible value and define $W_{r+1}$ to be the smallest subspace in $\Sigma$ which contains $U \cap \mathfrak{S}_{\mathfrak{D}_{j+1}}^{*}$. We claim $W_{r+1}$ is irreducible. For let $W_{r+1}=W^{\prime}+W^{\prime \prime}$ where $W^{\prime}, W^{\prime \prime}$ are two mutually orthogonal closed subspaces of $W_{r+1}$ which are both invariant under $\pi(G)$. Since $\operatorname{dim} W_{r+1} \cap \mathfrak{S}_{\mathfrak{D}_{j+1}}^{*} \geqq s>0$, at least one of the spaces $W^{\prime} \cap \mathfrak{S}_{\mathfrak{D}_{j+1}}^{*}, W^{\prime \prime} \cap \mathfrak{S}_{\mathfrak{D}_{j+1}}^{*}$ is not zero. Suppose $W^{\prime} \cap \mathfrak{S}_{\mathfrak{D}_{j+1}}^{*} \neq\{0\}$. Then $W^{\prime} \in \Sigma$ and in view of the definition of $s, s \leqq \operatorname{dim} W^{\prime} \cap \mathfrak{S}_{\mathfrak{D}_{j+1}^{*}}^{*}$. But $W^{\prime} \subset W_{r+1} \subset U$ and therefore

$$
s \leqq \operatorname{dim}\left(W^{\prime} \cap \mathfrak{S O D}_{j+1}\right) \leqq \operatorname{dim}\left(W_{r+1} \cap \mathfrak{S C}_{\mathfrak{D}_{j+1}}^{*}\right) \leqq \operatorname{dim}\left(U \cap \mathfrak{S D}_{j+1}^{*}\right)=s .
$$

Hence $W^{\prime} \cap \mathfrak{S}_{\mathfrak{D}_{j+1}}^{*}=W_{r+1} \cap \mathfrak{S}_{\mathfrak{D}_{j+1}}^{*}=U \cap \mathfrak{S}_{\mathfrak{D}_{j+1}}^{*}$ and so it follows from the definition of $W_{r+1}$ that $W_{r+1} \subset W^{\prime}$. This proves that $W^{\prime}=W_{r+1}$ and so $W_{r+1}$ is irreducible. 
Notice that $W_{r+1}=\{0\}$ if and only if $U_{r} \cap \mathfrak{S}_{\mathfrak{D}_{j+1}}^{*}=\{0\}$ and $W_{r+1} \neq\{0\}$ implies $W_{r+1} \cap \mathfrak{S}_{\mathfrak{D}_{j+1}}^{*} \neq\{0\}$. Since

$$
\operatorname{dim}\left(U_{r} \cap \mathfrak{S}_{\mathfrak{D}_{j+1}}^{*}\right)>\operatorname{dim}\left(U_{r+1} \cap \mathfrak{S}_{\mathfrak{D}_{j+1}}^{*}\right)
$$

unless $U_{r} \cap \mathfrak{S}_{\mathfrak{D}_{j+1}}^{*}=\{0\}$ and since $\operatorname{dim} \mathfrak{S}_{\mathfrak{D}_{j+1}}^{*}<\infty$, it follows that $W_{r}=\{0\}$ for $r$ sufficiently large. Let $r$ be the least integer $\geqq 0$ such that $W_{r+1}=\{0\}$. Then $U_{r} \cap \mathfrak{S}_{\mathfrak{D}_{j+1}}^{*}=\{0\}$ and therefore $V_{j, \mathscr{D}_{j+1}} \subset W_{1}+\cdots+W_{r}$. Now put $\mathfrak{S}_{j+1}=\mathfrak{S}_{j}+W_{1}+\cdots+W_{r}$. Then all the three conditions are fulfilled and the induction is therefore complete.

After this preparation we now come to the proof of the lemma. Let $\mathfrak{S}_{j+1}=\mathfrak{S}_{j}+\sum_{1 \leqq r \leqq s_{j}} W_{r}^{(j)}$ where $W_{r}^{(j)}$ are closed subspaces which are invariant and irreducible under $\pi(G)$ and which are orthogonal to $\mathfrak{S}_{j}$ and to each other. Then it is obvious that

$$
\mathfrak{S}_{i+1}=\sum_{0 \leqq i \leqq i} \sum_{1 \leqq r \leqq s_{i}} W_{r}^{(i)}
$$

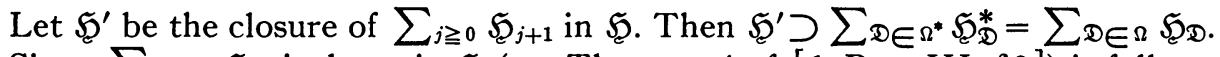
Since $\sum_{\mathfrak{D} \in \Omega} \mathfrak{S}_{\mathfrak{D}}$ is dense in $\mathfrak{T}$ (see Theorem 4 of [6, Part III, $\S 9$ ) it follows that $\mathfrak{S}=\mathfrak{S}^{\prime}$. Therefore $\mathfrak{S}$ is the closure of $\sum_{j \geqq 0} \sum_{1 \leqq r \leqq s_{j}} W_{r}^{(j)}$ and the lemma is proved.

LEMma 3. Let $\pi_{1}, \pi_{2}$ be two unitary representations of $G$ both satisfying the conditions of Lemma 1. Then if they have the same character they are equivalent.

Let $\mathfrak{S}_{i}$ be the representation space and $T_{\pi_{i}}$ the character of $\pi_{i}(i=1,2)$. Suppose $T_{\pi_{1}}=T_{\pi_{2}}=T$ (say). Then if $f \in C_{c}^{\infty}(G)$ and

$$
F(x)=\int \operatorname{conj}(f(y)) f(y x) d y,
$$

it is clear that $F \in C_{c}^{\infty}(G)$ and

$$
T(F)=\left\|\int f(x) \pi_{1}(x) d x\right\|^{2}=\left\|\int f(x) \pi_{2}(x) d x\right\|^{2} .
$$

Hence $T \neq 0$ unless $\pi_{i}(x)=0(i=1,2)$ for all $x \in G$. But since $\pi_{i}(1)$ is the unit operator on $\mathfrak{S}_{i}$, this is possible only if $\mathfrak{S}_{1}=\mathfrak{S}_{2}=\{0\}$. Since the lemma is true in this trivial case, we may assume that $T \neq 0$. Choose $f \in C_{c}^{\infty}(G)$ such that $T(f) \neq 0$. For any $z \in Z$ put $f_{z}(x)=f\left(z^{-1} x\right)(x \in G)$. Then

$$
T_{\pi}\left(f_{z}\right)=T_{\pi_{i}}\left(f_{z}\right)=\xi_{i}(z) T_{\pi_{i}}(f)=\xi_{i}(z) T(f) \quad(i=1,2)
$$

where $\pi_{i}(z)=\xi_{i}(z) \pi_{i}(1)$. Since $T(f) \neq 0$ it follows that $\xi_{1}(z)=\xi_{2}(z)$. Hence we can find a homomorphism $\eta$ of $K$ into $C$ such that $\eta(z)=\xi_{1}(z)=\xi_{2}(z)$ if $z \in Z \cap D$. Now define as above a representation $\pi_{i}^{*}$ of $K^{*}$ by putting $\pi_{i}^{*}\left(u^{*}\right)$ $=\eta\left(u^{-1}\right) \pi_{i}(u)(u \in K)$ and let $\mathfrak{S}_{i, \mathbb{D}}^{*}$ denote the subspace of those elements in 
$\mathfrak{S}_{i}$ which transform under $\pi_{i}^{*}\left(K^{*}\right)$ according to $\mathfrak{D}\left(\mathfrak{D} \in \Omega^{*}, i=1,2\right)$. Again we arrange the elements of $\Omega^{*}$ in a sequence $\mathfrak{D}_{j}(j \geqq 1)$. In view of Lemma 2 , $\mathfrak{S}_{i}$ can be written as a sum( $\left.{ }^{2}\right)$ of mutually orthogonal subspaces $W_{i, k} \neq\{0\}$ each of which is invariant and irreducible under $\pi_{i}$. Here $k$ runs over some subset $N_{i}$ of integers $(i=1,2)$. We shall now define a 1-1 mapping $\alpha$ of $N_{1}$ onto $N_{2}$ such that the representations of $G$ induced on $W_{1, k}$ and $W_{2, \alpha(k)}$ under $\pi_{1}$ and $\pi_{2}$ respectively are equivalent. This would prove the lemma.

For any $j$ let $N_{i}\left(\mathfrak{D}_{j}\right)$ denote the subset of $N_{i}$ consisting of those $k \in N_{i}$ for which $W_{i, k} \cap \mathfrak{L}_{i, \mathscr{D}_{j}}^{*} \neq\{0\}$. Since $\sum_{j \geqq 1}\left(W_{i, k} \cap \mathfrak{S}_{i, \mathscr{D}_{j}}^{*}\right)$ is dense in $W_{i, k}$ (Theorem 4 of $[6, \S 9]$ ) it follows that $\bigcup_{j \geqq 1} N_{i}\left(\mathfrak{D}_{j}\right)=N_{i}$. Put $M_{i, j}$ $=\bigcup_{1 \leqq r \leqq j} N_{i}\left(\mathfrak{D}_{r}\right)(j \geqq 1)$ and let $M_{i, 0}$ denote the empty set. We shall now define a 1-1 mapping $\alpha$ of $N_{1}$ onto $N_{2}$ with the following properties:

(i) $\alpha\left(N_{1}\left(\mathfrak{D}_{j}\right)\right)=N_{2}\left(\mathfrak{D}_{j}\right)(j \geqq 1)$.

(ii) The representations induced on $W_{1, k}$ and $W_{2, \alpha(k)}\left(k \in N_{1}\right)$ under $\pi_{1}$ and $\pi_{2}$ respectively are equivalent.

We proceed by induction on $j$. Suppose $\alpha$ has been defined as a 1-1 mapping $M_{1, r-1}$ onto $M_{2, r-1}(r \geqq 1)$ satisfying the above two requirements for $j \leqq r-1$ and $k \in M_{1, r-1}$. We shall now extend it on $M_{1, r}$. We may clearly assume that at least one of the above two sets $N_{1}\left(\mathfrak{D}_{r}\right), N_{2}\left(\mathfrak{O}_{r}\right)$ is not empty since otherwise $M_{i, r}=M_{i, r-1}(i=1,2)$ and no extension is needed. Let $P_{i, k}$ and $E_{i, \mathcal{D}}$ denote the orthogonal projections of $\mathfrak{S}_{i}$ on $W_{i, k}$ and $\mathfrak{S}_{i, \mathbb{D}}^{*}$ respectively $\left(\mathfrak{D} \in \Omega^{*}, i=1,2\right)$. Put

$$
\begin{aligned}
\phi_{i, k}(x) & =\operatorname{sp}\left(E_{i, D_{r}} P_{i, k} \pi_{i}(x) E_{i, D_{r}}\right), \\
\phi_{i}(x) & =\operatorname{sp}\left(E_{i, D_{r}} \pi_{i}(x) E_{i, D_{r}}\right) \quad\left(k \in N_{i}, \quad x \in G\right) .
\end{aligned}
$$

Then

$$
\phi_{i}=\sum_{k \in N_{i}\left(\mathscr{D}_{r}\right)} \phi_{i, k} \quad(i=1,2) .
$$

We claim $\phi_{1}=\phi_{2}$. For otherwise $\phi=\phi_{1}-\phi_{2} \neq 0$ and we can find a function $f \in C_{c}^{\infty}(G)$ such that $\int \phi(x) f(x) d x \neq 0$. Then as we have seen in the proof of Theorem 6 of [8], there exists a function $f^{\prime} \in C_{c}^{\infty}(G)$ such that

$$
E_{i, \mathcal{D r}} \int f(x) \pi_{i}(x) d x=\int f^{\prime}(x) \pi_{i}(x) d x
$$

Hence

$$
T_{\pi_{1}}\left(f^{\prime}\right)-T_{\pi_{2}}\left(f^{\prime}\right)=\int f(x) \phi(x) d x \neq 0
$$

which contradicts our hypothesis that $T_{\pi_{1}}=T_{\pi_{2}}$. Therefore

$$
\sum_{k \in N_{1}\left(\mathscr{D}_{r}\right)} \phi_{1, k}=\sum_{k \in N_{2}\left(\mathscr{D}_{r}\right)} \phi_{2, k}
$$


Since $W_{i, k} \cap \mathfrak{S}_{l, D_{r}}^{*} \neq\{0\}\left(k \in N_{i}\left(\mathfrak{D}_{r}\right)\right)$ it follows that $\phi_{i, k}(1) \neq 0$ for $k \in N_{i}\left(\mathfrak{D}_{r}\right)$. Now suppose $k \in N_{1}\left(\mathfrak{D}_{r}\right) \cap M_{1, r-1}$ so that $\alpha(k)$ has already been defined. Then by induction hypothesis, the representations induced on $W_{1, k}$ and $W_{2, \alpha(k)}$ are equivalent and therefore $\phi_{1, k}=\phi_{2, \alpha(k)}$. Since $\phi_{1, k}(1) \neq 0, \phi_{2, \boldsymbol{\alpha}(k)}(1) \neq 0$ and therefore $W_{2, \alpha(k)} \cap \mathfrak{S}_{2, \mathscr{D}_{r}}^{*} \neq\{0\}$. Hence $\alpha(k) \in N_{2}\left(\mathfrak{D}_{r}\right) \cap M_{2, r-1}$. Conversely suppose $l \in N_{2}\left(\mathfrak{N}_{r}\right) \cap M_{2, r-1}$. Since $\alpha$ is a 1-1 mapping of $M_{1, r-1}$ onto $M_{2, r-1}$ there is exactly one $k \in M_{1, r-1}$ such that $l=\alpha(k)$. Moreover the representations induced on $W_{1, k}$ and $W_{2, \alpha(k)}=W_{2, l}$ are equivalent. Therefore since $W_{2, l}$ $\cap \mathfrak{S}_{2, \mathscr{D}_{r}}^{*} \neq\{0\}$, it follows that $W_{1, k} \cap \mathfrak{S}_{1, \mathscr{D}_{r}}^{*} \neq\{0\}$. Hence $k \in N_{1}\left(\mathfrak{D}_{r}\right) \cap M_{1, r-1}$. This proves that $\alpha$ maps $N_{1}\left(\mathfrak{D}_{r}\right) \cap M_{1, r-1}$ onto $N_{2}\left(\mathfrak{D}_{r}\right) \cap M_{2, r-1}$. Let $N_{i}^{\prime}\left(\mathfrak{D}_{r}\right)$ be the complement of $N_{i}\left(\mathfrak{D}_{r}\right) \cap M_{i, r-1}$ in $N_{i}\left(\mathfrak{D}_{r}\right)(i=1,2)$. Then it is clear from what we have said that

$$
\sum_{k \in N_{1}^{\prime}\left(\mathscr{D}_{r}\right)} \phi_{1, k}=\sum_{k \in N_{2}^{\prime}\left(\mathcal{D}_{r}\right)} \phi_{2, k} .
$$

Let $\psi_{1}, \cdots, \psi_{s}$ be all the distinct functions among $\phi_{i, k}\left(k \in N_{i}^{\prime}\left(\mathfrak{D}_{r}\right), i=1,2\right)$. We know that none of these are zero and therefore from Theorem 1 of [8] it follows that each $\psi_{t}(1 \leqq t \leqq s)$ appears the same number of times in the two sums on either side of the above equation. This means that we can find a 1-1 mapping $k \rightarrow \alpha(k)$ of $N_{1}^{\prime}\left(\mathfrak{D}_{r}\right)$ onto $N_{2}^{\prime}\left(\mathfrak{D}_{r}\right)$ such that $\phi_{1, k}=\phi_{2, \alpha(k)} \neq 0$. In view of Theorem 1 of $[8]$ and Theorem 8 of $[6, \S 11]$, we can conclude that the representations induced on $W_{1, k}$ and $W_{2, \alpha(k)}\left(k \in N_{1}^{\prime}\left(\mathfrak{D}_{r}\right)\right)$ are equivalent. Thus $\alpha$ is now defined on $M_{r}$ and satisfies all the requirements. Therefore our induction is complete and the theorem follows.

Notice that if $k \in N_{i}(\mathfrak{D})\left(\mathfrak{D} \in \Omega^{*}\right)$, the representations induced on $W_{i, k}$ and $W_{i, l}$ cannot be equivalent unless $l \in N_{i}(\mathfrak{D})$. Hence if $\sigma$ is any unitary irreducible representation of $G$, there are only a finite number of values of $k$ such that the representation induced on $W_{i, k}$ is equivalent to $\sigma$. Let $n_{i}(\sigma)$ $(i=1,2)$ be this number. Then the above proof shows that $n_{1}(\sigma)=n_{2}(\sigma)$. In particular if $\pi_{1}=\pi_{2}=\pi$ (say), this number, which we now denote by $n(\sigma)$, is independent of the particular decomposition of $\mathfrak{S}$ into mutually orthogonal invariant irreducible subspaces. If $\omega$ is the equivalence class of $\sigma$, we call $n(\sigma)$ the multiplicity of $\omega$ in $\pi$. It is clear from Lemma 1 that for any $f$ $\in C_{c}^{\infty}(G)$,

$$
T_{\pi}(f)=\sum_{\omega \in \mathcal{E}} n(\omega) T_{\omega}(f)
$$

where $T_{\omega}$ is the character and $n(\omega)$ the multiplicity of $\omega$ in $\pi$ and the series is absolutely convergent. We may therefore write

$$
T_{\pi}=\sum_{\omega \in \mathcal{E}} n(\omega) T_{\omega} .
$$

Let $T$ be a distribution on $G$. We shall say that $T$ is a character of $G$ if there exists a representation $\pi$ satisfying the conditions of Lemma 1 such that 
$T$ is the character of $\pi$. $T$ is said to be unitary or irreducible if $\pi$ may be chosen to be unitary or irreducible.

3. Computation of some characters. We know that the mapping $(u, h, n)$ $\rightarrow$ uhn $\left(u \in K, h \in A_{+}, n \in N\right)$ is a homeomorphism of $K \times A_{+} \times N$ onto $G$. Since $Z \subset K, A_{+}$and $N$ are mapped isomorphically under the mapping $x \rightarrow x^{*}$ of $G$ on $G^{*}=G / D \cap Z$. Therefore we may identify $A_{+} N$ with its image under this mapping. Then $\left(u^{*}, h, n\right) \rightarrow u^{*} h n\left(u^{*} \in K^{*}, h \in A_{+}, n \in N\right)$ is a topological mapping of $K^{*} \times A_{+} \times N$ onto $G$. For any $x \in G$ put

$$
x^{*} u^{*}=u_{\star}^{*} h\left(x, u^{*}\right) n
$$

where $u_{x}^{*} \in K^{*}, h\left(x, u^{*}\right) \in A_{+}$, and $n \in N$. Then $u_{x}^{*}$ and $h\left(x, u^{*}\right)$ are continuous functions of $\left(x, u^{*}\right)$ on $G \times K^{*}$. Since $A_{+}$is simply connected, the mapping $H \rightarrow \exp H\left(H \in \mathfrak{h}_{\mathfrak{p}_{0}}\right)$ maps $\mathfrak{h}_{\mathfrak{p}_{0}}$ topologically onto $A_{+}$. We denote its inverse by $h \rightarrow \log h\left(h \in A_{+}\right)$. Put $H\left(x, u^{*}\right)=\log h\left(x, u^{*}\right)$. Finally let $\gamma\left(x, u^{*}\right)$ denote the unique element in $K$ such that

$$
u^{-1} x u \in \gamma\left(x, u^{*}\right) A_{+} N \quad\left(x \in G, u^{*} \in K^{*}\right) .
$$

Here $u$ is any element in $K$ lying $\left({ }^{3}\right)$ above $u^{*}$.

Normalise the Haar measure $d u^{*}$ on $K^{*}$ so that the total measure of $K^{*}$ is 1 . Let $\eta$ be a homomorphism of $K$ into $C$ and $\Lambda$ a (complex-valued) linear function on $\mathfrak{h}_{\mathfrak{p}_{0}}$. We regard the space $\mathfrak{S}=L_{2}\left(K^{*}\right)$ of all square-integrable functions on $K^{*}$ as a Hilbert space in the usual way and define a representation $\pi$ of $G$ on $\mathfrak{S}$ as follows. If $f \in \mathfrak{S}$ and $x \in G$,

$\pi(x) f\left(u^{*}\right)=\eta\left(\gamma\left(x^{-1}, u^{*}\right)\right) \exp \left\{-(\Lambda+2 \rho)\left(H\left(x^{-1}, u^{*}\right)\right)\right\} f\left(u_{x^{-1}}^{*}\right) \quad\left(u^{*} \in K^{*}\right)$.

Here $\pi(x) f\left(u^{*}\right)$ denotes the value of the function $\pi(x) f$ at $u^{*}$ and $\rho$ has the same meaning as in $[6, \S 12]$. It is easy to verify (see $[6, \S 12]$ ) that $\pi$ is in fact a representation.

Let $\mathfrak{m}_{0}, \mathfrak{h}_{\mathfrak{t}_{0}}$, and $\mathfrak{h}_{0}$ be the subalgebras of $\mathfrak{g}_{0}$ as defined in $[6, \S 2]$ and let $M_{0}, A_{-}^{0}$, and $A^{0}$ be the corresponding analytic subgroups of $G$. Then $A^{0}$ is a maximal connected abelian subgroup of $G$ and therefore it is closed. Let $M$ and $A_{-}$respectively be the centralizers of $A_{+}$and $A^{0}$ in $K$. Then they are both closed subgroups of $K$. Since $\mathfrak{m}_{0}$ and $\mathfrak{h}_{\mathfrak{t}_{0}}$ respectively are the centralizers of $\mathfrak{h}_{\mathfrak{p}_{0}}$ and $\mathfrak{h}_{0}$ in $\mathfrak{f}_{0}$ (see Lemma $4, \S 2$ of [6]), $M_{0}$ and $A_{-}^{0}$ are the components of identity of $M$ and $A_{-}$respectively. Put $A=A_{+} A_{-}$. We shall see later that $A$ is exactly the centralizer of $A^{0}$ in $G$.

Lemma 4. Let $m$ be an element in $M$. Then $m N m^{-1}=N$.

Let $\mathfrak{g}$ be the complexification of $\mathfrak{g}_{0}$ and $\mathfrak{h}, \mathfrak{h}_{\mathfrak{p}}, \mathfrak{h}_{\mathfrak{t}}, \mathfrak{m}$ the subalgebras of $\mathfrak{g}$ spanned by $\mathfrak{h}_{0}, \mathfrak{h}_{\mathfrak{p}_{0}}, \mathfrak{h}_{\mathfrak{f}_{0}}, \mathfrak{m}_{0}$ respectively over $C$. We define positive roots of $\mathfrak{g}$

(3) Let $V$ be the space of all cosets $x B(x \in G)$ with respect to a closed subgroup $B$ of $G$ Then we say that $x$ lies above $v(x \in G, v \in V)$ if $x$ lies in the coset $v$. 
(with respect to $\mathfrak{h}$ ) and divide them into two disjoint classes $P_{+}$and $P_{-}$as described in $[6, \S 2]$. For every root $\alpha$ select an element $X_{\alpha} \neq 0$ in $g$ such that $\left[H, X_{\alpha}\right]=\alpha(H) X_{\alpha}(H \in \mathfrak{h})$. Then if $\mathfrak{n}=\sum_{\alpha \in P_{+}} C X_{\alpha}$ and $\mathfrak{n}^{-}=\sum_{\alpha \in P_{+}} C X_{-\alpha}$ we have $\mathfrak{g}=\mathfrak{h}_{\mathfrak{p}}+\mathfrak{m}+\mathfrak{n}+\mathfrak{n}^{-}$. Let $x \rightarrow$ Ad $(x)$ denote the adjoint representation of $G$ on g. Then if $m \in M, \operatorname{Ad}(m) H=H$ and therefore $\left[H, \operatorname{Ad}(m) X_{\alpha}\right]$ $=\alpha(H) \operatorname{Ad}(m) X_{\alpha}$ for all $H \in \mathfrak{h}_{\mathfrak{p}}$ and $\alpha \in P_{+}$. Since $\left[\mathfrak{h}_{\mathfrak{p}}, \mathfrak{m}\right]=\{0\}$ it follows from the above decomposition of $\mathfrak{g}$ that $\operatorname{Ad}(m) X_{\alpha} \in \mathfrak{n}\left(\alpha \in P_{+}\right)$. Hence $\operatorname{Ad}(m) \mathfrak{n}=\mathfrak{n}$ and therefore $m N m^{-1}=N$.

Let $M^{*}$ denote the image of $M$ in $K^{*}$. Then $M^{*}$ is the centralizer of $A_{+}$ in $K^{*}$, and therefore it is closed and hence compact. Let $M_{1}$ be any subgroup of $M$ containing $M_{0} Z$. Let $M_{1}^{*}$ and $M_{0}^{*}$ denote the images of $M_{1}$ and $M_{0}$ respectively in $K^{*}$. Then $M_{0}^{*}$ is the connected component of $M^{*}$ and therefore $M^{*} / M_{0}^{*}$ is both compact and discrete and hence finite. From this it follows that $M_{1}^{*}$ is compact. We normalise the Haar measure $d m^{*}$ on $M_{1}^{*}$ so as to make the total measure of $M_{1}^{*}$ equal to 1 .

Let $\tau$ denote the right regular representation of $K^{*}$ on $\mathfrak{S}$ so that $\tau\left(v^{*}\right) f\left(u^{*}\right)$ $=f\left(u^{*} v^{*}\right)\left(u^{*}, v^{*} \in K^{*}, f \in \mathfrak{S}\right)$. Then it follows easily from Lemma 4 that $\tau\left(m^{*}\right)$ commutes with $\pi(x)$ if $x \in G$ and $m^{*} \in M^{*}$. Put

$$
T=\int f(x) \pi(x) d x, \quad S=\int_{M_{1}^{*}} g\left(m^{*}\right) \tau\left(m^{*}\right) d m^{*}
$$

where $f \in C_{c}^{\infty}(G)$ and $g$ is a continuous function on $M_{1}^{*}$. Now if $v \in K$,

$$
\pi(v) \phi\left(u^{*}\right)=\eta\left(v^{-1}\right) \phi\left(v^{*-1} u^{*}\right) \quad\left(\phi \in \mathfrak{S}, u^{*} \in K^{*}\right) .
$$

Therefore it follows from the Peter-Weyl Theorem for $K^{*}$ that no irreducible representation of $K$ occurs more often in the reduction of $\pi(K)$ than its degree. Hence Lemma 1 is applicable. Since $S$ is a bounded operator it follows (see Lemma 1 of [8]) that $T S$ is of the trace class. We propose to compute Sp $(T S)$.

Let $\phi$ be a continuous function on $K^{*}$. For a fixed $m^{*}$ in $M_{1}^{*}$ put $\phi^{\prime}$ $=\tau\left(m^{*}\right) \phi$. Then

$$
T \phi^{\prime}\left(u^{*}\right)=\int f(x) \pi(x) \phi^{\prime}\left(u^{*}\right) d x=\int f\left(u x^{-1}\right) \pi\left(u x^{-1}\right) \phi^{\prime}\left(u^{*}\right) d x
$$

where $u$ is some element in $K$ lying above $u^{*}$. Let $d v, d h, d n$ denote the Haar measures on $K, A_{+}$, and $N$ respectively. We normalise $d v$ in such a way that for any continuous function $\psi$ on $K$ which vanishes outside a compact set,

$$
\int_{K} \psi(v) d v=\int_{K^{*}} \psi^{*}\left(v^{*}\right) d v^{*}
$$

where $\psi^{*}\left(v^{*}\right)=\sum_{\gamma \in D \cap Z} \psi(v \gamma)(v \in K)$. Moreover we assume that $d x, d h$, and $d n$ are so normalised that 


$$
d x=\exp \{2 \rho(\log h)\} d v d h d n \quad\left(x=v h n ; v \in K, h \in A_{+}, n \in N\right)
$$

(see Lemma 35 of $[6, \S 12])$. Then

$$
\begin{aligned}
T \phi^{\prime}\left(u^{*}\right) & =\int f\left(u(v h n)^{-1}\right) \eta\left(u^{-1} v\right) \phi^{\prime}\left(v^{*}\right) \exp \{\Lambda(\log h)\} d v d h d n \\
& =\int f\left(u(h n)^{-1} v^{-1}\right) \eta\left(u^{-1} v\right) \phi\left(v^{*} m^{*}\right) \exp \{\Lambda(\log h)\} d v d h d n .
\end{aligned}
$$

Now since $A_{+}$is abelian and $N$ is nilpotent, they are both unimodular. Hence

$$
\int f\left(u(h n)^{-1} v^{-1}\right) d h d n=\int f\left(u n h v^{-1}\right) d h d n .
$$

But $n h=h\left(h^{-1} n h\right)$ and for a fixed $h, d\left(h^{-1} n h\right)=\exp \{-2 \rho(\log h)\} d n$ as follows easily from Lemma 5 of $[6, \S 2]$. Therefore

$$
\int f\left(u(h n)^{-1} v^{-1}\right) d h d n=\int f\left(u h n v^{-1}\right) \exp \{2 \rho(\log h)\} d h d n
$$

and

$$
T S \phi\left(u^{*}\right)=\int f\left(u h n v^{-1}\right) \eta\left(u^{-1} v\right) \phi\left(v^{*} m^{*}\right) g\left(m^{*}\right) \exp \{(\Lambda+2 \rho)(\log h)\} d v d h d n d m^{*} .
$$

Put

$$
F(u, v)=\int f\left(u h n v^{-1}\right) \eta\left(u^{-1} v\right) \exp \{(\Lambda+2 \rho)(\log h)\} d h d n \quad(u, v \in K) .
$$

Since $f$ vanishes outside a compact set it is clear that for a fixed $v, F(u, v)$ vanishes outside a compact set on $K$. Since $F(u, v \gamma)=F\left(u \gamma^{-1}, v\right)(\gamma \in Z)$ it follows that the sum $\sum_{\gamma \in Z \cap D} F(u, v \gamma)$ is defined and depends only on $\left(u^{*}, v^{*}\right)$. Put

$$
F\left(u^{*}, v^{*}\right)=\sum_{\gamma \in z \cap_{D}} F(u, v \gamma) .
$$

Then it is seen without difficulty that $F^{*}$ is an indefinitely differentiable function on $K^{*} \times K^{*}$ and

$$
\begin{aligned}
T S \phi\left(u^{*}\right) & =\int F^{*}\left(u^{*}, v^{*}\right) \phi\left(v^{*} m^{*}\right) g\left(m^{*}\right) d v^{*} d m^{*} \\
& =\int F^{*}\left(u^{*}, v^{*} m^{*-1}\right) \phi\left(v^{*}\right) g\left(m^{*}\right) d v^{*} d m^{*} \\
& =\int \Phi\left(u^{*}, v^{*}\right) \phi\left(v^{*}\right) d v^{*}
\end{aligned}
$$


where

$$
\Phi\left(u^{*}, v^{*}\right)=\int_{M_{1}^{*}} F^{*}\left(u^{*}, v^{*} m^{*-1}\right) g\left(m^{*}\right) d m^{*} .
$$

Thus $T S$ is represented here as an integral operator with the kernel $\Phi$. It is clear that $\Phi$ is also indefinitely differentiable on $K^{*} \times K^{*}$. In order to compute Sp $T S$ we make use of the following lemma.

Lemma 5. Let $\lambda\left(u^{*}, v^{*}\right)$ be an indefinitely differentiable function on $K^{*} \times K^{*}$ and let $L$ be the bounded linear operator on $L_{2}\left(K^{*}\right)$ defined by

$$
L \phi\left(u^{*}\right)=\int \lambda\left(u^{*}, v^{*}\right) \phi\left(v^{*}\right) d v^{*} \quad\left(\phi \in L_{2}\left(K^{*}\right)\right) .
$$

Then if $\left.{ }^{4}\right) L$ is of the trace class

$$
\operatorname{sp} L=\int_{K^{*}} \lambda\left(u^{*}, u^{*}\right) d u^{*}
$$

As above let $\Omega^{*}$ denote the set of all equivalence classes of simple finitedimensional representations of $K^{*}$. For every $\mathfrak{D} \in \Omega^{*}$ choose a unitary matrix representation $\sigma^{\mathfrak{D}}$ in $\mathfrak{D}$. Let $d(\mathfrak{D})$ denote the degree and $\sigma_{1 j}^{\mathfrak{D}}, 1 \leqq i, j$ $\leqq d(\mathfrak{D})$, the matrix coefficients of $\sigma^{\mathfrak{D}}$. Then the functions $d(\mathfrak{D})^{1 / 2} \sigma_{i j}^{\mathscr{D}}, 1 \leqq i, j$ $\leqq d(\mathfrak{D})\left(\mathfrak{D} \in \Omega^{*}\right)$, form a complete orthonormal set in $L_{2}\left(K^{*}\right)$. Hence if $L$ is of the trace class,

$$
\begin{aligned}
\operatorname{sp} L & =\sum_{\mathfrak{D} \in \Omega^{*}} d(\mathfrak{D})\left(\underset{\sigma_{i j}}{\mathfrak{D}}, \underset{\sigma_{i j}}{\mathfrak{D}}\right) \\
& =\sum_{\mathfrak{D} \in \Omega^{*}} d(\mathfrak{D}) \int_{K^{*}} \chi \mathscr{D}\left(u^{*-1} v^{*}\right) \lambda\left(u^{*}, v^{*}\right) d u^{*} d v^{*}
\end{aligned}
$$

where $\chi_{D}$ is the character of the class $\mathfrak{D}$ and $(\psi, \phi)$ denotes the usual scalar product in $L_{2}\left(K^{*}\right)\left(\psi, \phi \in L_{2}\left(K^{*}\right)\right)$. Put

$$
\lambda_{0}\left(v^{*}\right)=\int \lambda\left(u^{*}, u^{*} v^{*}\right) d u^{*}
$$

Then $\lambda_{0}\left(v^{*}\right)$ is an idefinitely differentiable function on $K^{*}$ and

$$
\operatorname{sp} L=\sum_{\mathfrak{D} \in \Omega^{*}} d(\mathfrak{D}) \int \chi \mathfrak{D}\left(v^{*}\right) \lambda_{0}\left(v^{*}\right) d v^{*} .
$$

Let $\mathfrak{S}$ be the Banach space of continuous functions $\phi$ on $K^{*}$ with the norm

(4) It is not difficult to show by the method of $\$ 5$ of [8] that $L$ is in fact of the trace class. However we do not need this fact here. 
$|\phi|=\sup _{K^{*}}\left|\phi\left(v^{*}\right)\right|$. For $u^{*} \in K^{*}$ let $l\left(u^{*}\right)$ denote the bounded linear operator on $\mathfrak{S}$ given by

$$
l\left(u^{*}\right) \phi\left(v^{*}\right)=\phi\left(u^{*-1} v^{*}\right) \quad\left(\phi \in \mathfrak{S}, v^{*} \in K^{*}\right) .
$$

Then $u^{*} \rightarrow l\left(u^{*}\right)$ is a representation of $K^{*}$ on $\mathfrak{S}$. Since $\lambda_{0}$ is of class $C^{\infty}$ it is differentiable under $l$ (see $[6, \S 9])$. Therefore if we use the arguments of the proof of Lemma 3 of [8] we see that the series $\sum_{\mathscr{D} \in \Omega^{*}} d(\mathfrak{D}) \chi_{\mathfrak{D}}\left(v^{*-1}\right) l\left(v^{*}\right) \lambda_{0} d v^{*}$ converges absolutely in $\mathfrak{S}$ to $\lambda_{0}$. Hence

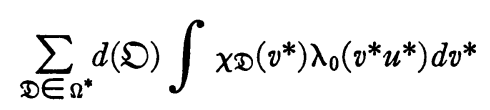

converges uniformly to $\lambda_{0}\left(u^{*}\right)$ on $K^{*}$. Putting $u^{*}=1^{*}$ we get

$$
\text { sp } L=\lambda_{0}\left(1^{*}\right)=\int \lambda\left(u^{*}, u^{*}\right) d u^{*} \text {. }
$$

If we apply the above lemma to the operator $T S$ we get

$$
\text { sp } T S=\int \Phi\left(u^{*}, u^{*}\right) d u^{*}=\int F^{*}\left(u^{*}, u^{*} m^{*-1}\right) g\left(m^{*}\right) d u^{*} d m^{*} .
$$

Now $M_{1}$ is the complete inverse image of $M_{1}^{*}$ in $K$. Therefore it is closed. We normalise the Haar measure $d m$ on $M_{1}$ in such a way that for any continuous function $\alpha$ on $M_{1}$ which vanishes outside a compact set,

$$
\int_{M_{1}} \alpha(m) d m=\int_{M_{1}^{*}} \alpha^{*}\left(m^{*}\right) d m^{*}
$$

where $\alpha^{*}\left(m^{*}\right)=\sum_{\gamma \in Z \cap D} \alpha(m \gamma)\left(m \in M_{1}\right)$. Then if we recall the definition of $F^{*}$ we find that

$$
\begin{aligned}
\int F^{*}\left(u^{*}, u^{*} m^{*-1}\right) g\left(m^{*}\right) d m^{*} & \\
= & \int F\left(u, u m^{-1}\right) g\left(m^{*}\right) d m \\
& =\int f\left(u h n m u^{-1}\right) \eta\left(m^{-1}\right) g\left(m^{*}\right) \exp \{(\Lambda+2 \rho)(\log h)\} d h d n d m
\end{aligned}
$$

where $u$ is any element in $K$ lying above $u^{*}$. Now $h n m=m h\left(m^{-1} n m\right)$ and for fixed $m, d\left(m^{-1} n m\right)=d n$. Hence

$$
\Phi\left(u^{*}, u^{*}\right)=\int f\left((m h n) u^{*}\right) \eta\left(m^{-1}\right) g\left(m^{*}\right) \exp \{(\Lambda+2 \rho)(\log h)\} d h d n d m
$$

(where $x^{u *}=u x u^{-1}$ ) and therefore 
sp $T S=\int f\left((m h n)^{u^{*}}\right) \eta\left(m^{-1}\right) g\left(m^{*}\right) \exp \{(\Lambda+2 \rho)(\log h)\} d u^{*} d h d n d m$.

Now let $m_{1} \in M_{1}$. Then

$$
\left(m_{1} m m_{1}^{-1} h n\right)^{u^{*}}=\left(m h n^{\prime}\right)^{u^{*} m_{\mathbf{i}}^{*}}
$$$$
\left(m \in M_{1}, h \in A_{+}, n \in N\right)
$$

where $n^{\prime}=m_{1}^{-1} n m_{1} \in N$. From this it follows that

$$
\text { sp } T S=\text { sp } T S^{\prime}
$$

where $S^{\prime}=\int_{M_{1}^{*}} g^{\prime}\left(m^{*}\right) \tau\left(m^{*}\right) d m^{*}$ and $g^{\prime}\left(m^{*}\right)=g\left(\left(m_{1} m m_{1}^{-1}\right)^{*}\right)\left(m \in M_{1}\right)$. Hence it is clear that

$$
\text { sp } T S=\int f\left((m h n)^{u^{*}}\right) \eta\left(m^{-1}\right) \xi^{*}\left(m^{*}\right) \exp \{(\Lambda+2 \rho)(\log h)\} d u^{*} d h d n d m
$$

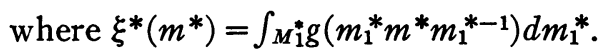

Now let $\sigma$ be an irreducible unitary matrix representation of $M_{1}^{*}$ of degree $d$ and let $\sigma_{i j}, 1 \leqq i, j \leqq d$, denote its matrix coefficients. Put

$$
E_{i j}=d \int_{M_{1}^{*}} \sigma_{i j}\left(m^{*-1}\right) \tau\left(m^{*}\right) d m^{*}, \quad 1 \leqq i, j \leqq d,
$$

and $E_{i}=E_{i i}(1 \leqq i \leqq d)$. Then $E_{i}$ are mutually orthogonal projections which commute with $\pi(x)(x \in G)$. Hence if $\mathfrak{S}=L_{2}\left(K^{*}\right), \mathfrak{S}_{i}=E_{i} \mathfrak{S}, 1 \leqq i \leqq d$, are closed subspaces which are invariant under $\pi(G)$. Let $\pi_{i}$ be the representation of $G$ induced on $\mathfrak{S}_{i}$ under $\pi$. Then it is clear that the operator $\int f(x) \pi_{i}(x) d x$ is of the trace class and

$$
\operatorname{sp}\left(\int f(x) \pi_{i}(x) d x\right)=\operatorname{sp} T E_{i}
$$

But

$$
d \int_{M_{1}} \sigma_{i i}\left(m_{1}^{*} m^{*} m_{1}^{*-1}\right) d m_{1}^{*}=\xi^{*}\left(m^{*}\right) \quad\left(m^{*} \in M_{1}^{*}\right)
$$

where $\xi^{*}$ is the character of $\sigma$. Hence

$$
\begin{aligned}
\operatorname{sp}\left(\int f(x) \pi_{i}(x) d x\right)=\int f\left((m h n)^{*}\right) \eta\left(m^{-1}\right) \xi^{*}\left(m^{*-1}\right) \\
\cdot \exp \{(\Lambda+2 \rho)(\log h)\} d u^{*} d h d n d m .
\end{aligned}
$$

Let $\sigma^{\prime}$ be the representation of $M_{1}^{*}$ contragredient to $\sigma$. Define a representation $\sigma^{\prime \prime}$ of $M_{1}$ as follows:

$$
\sigma^{\prime \prime}(m)=\eta\left(m^{-1}\right) \sigma^{\prime}\left(m^{*}\right) \quad\left(m \in M_{1}\right) .
$$

Let $\delta$ be the equivalence class and $\xi_{\delta}$ the character of $\sigma^{\prime \prime}$. Then 


$$
\xi_{\delta}(m)=\eta\left(m^{-1}\right) \xi^{*}\left(m^{*-1}\right)
$$

Hence $\left({ }^{5}\right)$

$$
\operatorname{sp}\left(\int f(x) \pi_{i}(x) d x\right)=T_{\Lambda, \delta}(f)
$$

where

$$
T_{\Delta, \delta}(f)=\int f\left((m h n) u^{*}\right) \xi_{\delta}(m) \exp \{(\Lambda+2 \rho)(\log h)\} d u^{*} d h d n d m .
$$

Let $\omega_{M_{1}}$ be the set of all equivalence classes of finite-dimensional simple representations of $M_{1}$. Then we have proved the following theorem.

THEOREM 1. Let $\Lambda$ be a linear function on $\mathfrak{h}_{\mathfrak{p}_{0}}$ and $\delta$ a class in $\omega_{M_{1}}$. Let $\xi_{\delta}$ denote the character of $\delta$. Let $T_{\Lambda, \delta}$ denote the distribution given by

$$
T_{\Delta, \delta}(f)=\int_{M_{1}} \xi_{\delta}(m) d m \int f\left((m h n) u^{*}\right) \exp \{(\Lambda+2 \rho)(\log h)\} d u^{*} d h d n
$$

$\left(f \in C_{c}^{\infty}(G)\right)$. Then $T_{\Lambda, \delta}$ is a character of $G$.

The above formula shows that $T_{\Lambda, \delta}$ is not only a distribution but actually a measure (see [10]). Therefore it may be regarded as a continuous linear functional on the space $C_{c}(G)$ of all continuous functions on $G$ which vanish outside a compact set. Moreover we know (see $[6, \S 12]$ ) that if $\Lambda+\rho$ takes pure imaginary values on $\mathfrak{h}_{\mathfrak{p}_{0}}$ and $|\eta(x)|=1(x \in G), \pi$ (and therefore $\left.\pi_{i}\right)$ is a unitary representation of $G$. Hence $T_{\Lambda, \delta}$ is a unitary character if $\Lambda+\rho$ is pure imaginary on $\mathfrak{h}_{\mathfrak{p}_{0}}$ and $\delta$ is a unitary class (i.e. the class of a unitary representation of $\left.M_{1}\right)$. For any $\mathfrak{D} \in \Omega$ let $(\mathfrak{D}: \delta)$ denote the number of times $\delta$ occurs in the reduction of $\mathfrak{D}$ with respect to $M_{1}$. Then we know (see A. Weil [12, p. 83] and $[6, \S 12])$ that $\mathfrak{D}$ occurs exactly $(\mathfrak{D}: \delta)$ times in the reduction of $\pi_{i}$ with respect to $K$.

We shall now derive another expression for the character $T_{\Lambda, \delta}$ when $M_{1}=M_{0} Z$. Since $Z \subset K$, every element $h \in A^{0} Z$ can be written uniquely as $h=h_{+} h_{-}$where $h_{+} \in A_{+}$and $h_{-} \in A_{-}^{0} Z$. Let $A_{-}^{0 *}$ be the image of $A_{-}^{0}$ in $K^{*}$. Then $A_{-}^{0 *}$ is a maximal abelian subgroup of the compact Lie group $M_{0}^{*}$. Hence every element in $M_{0}^{*}$ is conjugate (with respect to $M_{0}^{*}$ ) to some element in $A_{-}^{0 *}$. Therefore it follows from known theory (see Weyl [13]) that if the Haar measure $d h_{-}$on $A_{-}^{0} Z$ is suitably normalised

$$
\int_{M_{0} Z} \gamma(m) d m=\int_{A_{-}^{0} Z} \gamma\left(h_{-}\right) \Delta_{-}\left(h_{-}\right)^{2} d h_{-}
$$

(5) Since $E_{j i} E_{i}=E_{j}$ and $E_{i j} E_{j}=E_{i}$ and $E_{i j}$ commutes with $\pi(x)$, it is clear that $\pi_{i}$ and $\pi_{j}$ are equivalent under the mapping $\psi \rightarrow E_{j i} \psi\left(\psi \in \mathfrak{W}_{i}\right.$ ) of $\mathfrak{W}_{i}$ onto $\mathfrak{W}_{i}$. 
for any continuous class function $\gamma$ on $M_{0} Z$ which vanishes outside a compact set. Here

$$
\Delta_{-}\left(h_{-}\right)=\left|\prod_{\alpha \in P_{-}}\left(e^{\alpha(H) / 2}-e^{-\alpha(H) / 2}\right)\right|
$$

where $H$ is any element in $\mathfrak{h}_{\mathfrak{t}_{0}}$ such that $h_{-}^{-1} \exp H \in Z$. If we put

$$
\gamma(m)=\xi_{\delta}(m) \int f\left((m h n)^{u^{*}}\right) \exp \{(\Lambda+2 \rho)(\log h)\} d u^{*} d h d n
$$

we get the following result.

LeMma 6. It is possible to normalize the Haar measures $d h_{-}$and $d h_{+}$on $A_{-}^{0} Z$ and $A_{+}$in such a way that

$$
\begin{array}{r}
T_{\Lambda, \delta}(f)=\int_{A_{-}^{0} \boldsymbol{0}} \xi_{\delta}\left(h_{-}\right) \Delta_{-}\left(h_{-}\right)^{2} d h_{-} \int f\left(\left(h_{-} h_{+} n\right)^{u^{*}}\right) \\
\cdot \exp \left\{(\Lambda+2 \rho)\left(\log h_{+}\right)\right\} d u^{*} d h_{+} d n
\end{array}
$$

for every linear function $\Lambda$ on $\mathfrak{h}_{\mathfrak{p}_{0}}, \delta \in \omega_{M_{0} z}$ and $f \in C_{c}^{\infty}(G)$.

4. Transformation of certain integrals. We keep to the notation of $\$ 2$. Let $X \rightarrow \operatorname{ad} X\left(X \in \mathfrak{g}_{0}\right)$ denote the adjoint representation of $\mathfrak{g}_{0}$. We say that an element $X \in \mathfrak{g}_{0}$ is singular if the characteristic polynomial of ad $X$ in the indeterminate $\lambda$ is divisible by $\lambda^{l+1}\left(l=\operatorname{dim} \mathfrak{h}_{0}\right)$. The coefficient of $\lambda^{l}$ is clearly a polynomial function $\left(^{6}\right) F(X)$ on $\mathfrak{g}_{0}$ which is not identically zero. Since $X$ is singular if and only if $F(X)=0$, it follows that the set of singular elements is closed and nowhere dense in the Euclidean space $g_{0}$. We call an element regular if it is not singular. Let $x \rightarrow \operatorname{Ad}(x)(x \in G)$ denote the adjoint representation of $G$ on $\mathfrak{g}_{0}$. It is clear that $\operatorname{Ad}(x) X(x \in G)$ is regular if and only if $X$ is regular.

Leмma 7. Let $H$ be a regular element in $\mathfrak{h}_{0}$. Suppose $x$ is an element in $G$ such that $\operatorname{Ad}(x) H \in \mathfrak{h}_{0}$. Then $x \in A_{-}^{\prime} A_{+}$where $A_{-}^{\prime}$ is the normalizer of $A^{0}$ in $K$.

Since $H$ is regular, $\mathfrak{h}_{0}$ is the centralizer of $H$ in $\mathfrak{g}_{0}$. Hence Ad $(x) \mathfrak{h}_{0}$ is the centralizer of Ad $(x) H$. But since Ad $(x) H \in \mathfrak{h}_{0}, \mathfrak{h}_{0}$ is contained in this centralizer and therefore $\mathfrak{h}_{0} \subset \operatorname{Ad}(x) \mathfrak{h}_{0}$. Since $\mathfrak{h}_{0}$ and $\operatorname{Ad}(x) \mathfrak{h}_{0}$ have the same dimension, $\mathfrak{h}_{0}=\operatorname{Ad}(x) \mathfrak{h}_{0}$. Let $x=u h n^{\prime}\left(u \in K, h \in A_{+}, n^{\prime} \in N\right)$. Since $h n^{\prime}=n h$ where $n=h n^{\prime} h^{-1} \in N$ we get

$$
\operatorname{Ad}\left(u^{-1}\right) \mathfrak{h}_{0}=\operatorname{Ad}(n h) \mathfrak{h}_{0}=\operatorname{Ad}(n) \mathfrak{h}_{0} \subset \mathfrak{h}_{0}+\mathfrak{n}_{0} .
$$

On the other hand Ad $\left(u^{-1}\right) \mathfrak{h}_{\mathfrak{p}_{0}} \subset \mathfrak{p}_{0}$ and $\mathfrak{p}_{0} \cap\left(\mathfrak{h}_{0}+\mathfrak{n}_{0}\right)=\mathfrak{h}_{\mathfrak{p}_{0}}$ where $\mathfrak{p}_{0}$ is defined

(6) Let $V$ be a vector space over $R$ or $C$. A complex-valued function $f$ on $V$ is called a polynomial function if it can be written as a polynomial (with complex coefficients) in linear functions on $V$. 
as in [6, §2]. Similarly Ad $\left(u^{-1}\right) \mathfrak{h}_{\mathfrak{t}_{0}} \subset \mathfrak{f}_{0}$ and $\mathfrak{f}_{0} \cap\left(\mathfrak{h}_{0}+\mathfrak{n}_{0}\right)=\mathfrak{h}_{\mathfrak{t}_{0}}$. Therefore

$$
\text { Ad }\left(u^{-1}\right) \mathfrak{h}_{\mathfrak{p}_{0}} \subset \mathfrak{h}_{\mathfrak{p}_{0}}, \quad \text { Ad }\left(u^{-1}\right) \mathfrak{h}_{\mathfrak{t}_{0}} \subset \mathfrak{h}_{\mathfrak{t}_{0}} \text {. }
$$

Hence $\operatorname{Ad}\left(u^{-1}\right) \mathfrak{h}_{0} \subset \mathfrak{h}_{0}$ and so $u \in A_{-}^{\prime}$. Therefore in order to prove our assertion we have only to show that $n=1$. This follows from the lemma below.

Lemma 8. Let $H$ be a regular element in $\mathfrak{h}_{0}$. Then $n \rightarrow \operatorname{Ad}(n) H(n \in N)$ is a 1-1 mapping of $N$ onto the set of all elements of the form $H+Z\left(Z \in \mathfrak{n}_{0}\right)$.

Define $X_{\alpha}$ and $\mathfrak{n}$ as in the proof of Lemma 4. Then $\mathfrak{n}_{0}=\mathfrak{n} \cap \mathfrak{g}_{0}$; since $N$ is nilpotent every $n \in N$ can be written in the form $n=\exp X\left(X \in \mathfrak{n}_{0}\right)$. Therefore Ad $(n) H-H=\exp (\operatorname{ad} X) H-H \in \mathfrak{n}_{0}$ since $\left[\mathfrak{n}_{0}, \mathfrak{h}_{0}\right] \subset \mathfrak{n}_{0}$. This proves that $\operatorname{Ad}(n) H=H+Z$ where $Z \in \mathfrak{n}_{0}$. Now suppose $\operatorname{Ad}(n) H=H$. Then $\exp (\operatorname{ad} X) H$ $-H=0$ and this implies that $X=0$. For otherwise suppose $X \neq 0$. Then $X=\sum_{\alpha \in P_{+}} a_{\alpha} X_{\alpha}\left(a_{\alpha} \in C\right)$ and not all $a_{\alpha}$ are zero. Let $\beta$ be the lowest root in $P_{+}$such that $a_{\beta} \neq 0$. Then

$$
\exp (\operatorname{ad} X) H-H \equiv(\operatorname{ad} X) H \equiv-a_{\beta} \beta(H) X_{\beta} \bmod \sum_{\alpha>\beta} C X_{\alpha} .
$$

Since $H$ is regular, $\beta(H) \neq 0$ and therefore it follows from the linear independence of $X_{\alpha}\left(\alpha \in P_{+}\right)$over $C$ that $\exp (\operatorname{ad} X) H-H \neq 0$ in contradiction with our hypothesis. Now if $\operatorname{Ad}\left(n_{1}\right) H=\operatorname{Ad}\left(n_{2}\right) H\left(n_{1}, n_{2} \in N\right), \operatorname{Ad}\left(n_{2}^{-1} n_{1}\right) H$ $=H$ and therefore $n_{2}^{-1} n_{1}=1$ so that $n_{1}=n_{2}$.

Finally we claim that every element of the form $H+Z\left(Z \in \mathfrak{n}_{0}\right)$ can be written as $\operatorname{Ad}(n) H$ for some $n \in N$. For otherwise choose $Z$ such that such a representation is impossible. Then clearly $Z \neq 0$. Let

$$
Z=a_{\alpha} X_{\alpha}+\sum_{\beta>\alpha} a_{\beta} X_{\beta} \quad\left(a_{\alpha}, a_{\beta} \in C\right)
$$

where $\alpha$ is a root in $P_{+}$and $a_{\alpha} \neq 0$. We choose $Z$ in such a way that $\alpha$ has the highest possible value. Since $H$ is regular the mapping $X \rightarrow[H, X]\left(X \in \mathfrak{n}_{0}\right)$ is a nonsingular linear mapping of $\mathfrak{n}_{0}$ into itself. Hence there exists a $Y \in \mathfrak{n}_{0}$ such that $[H, Y]=Z$. Put $n_{1}=\exp Y$. Then it is clear that

$$
\text { Ad } \begin{aligned}
\left(n_{1}\right)(H+Z)-H & \equiv[Y, H]+Z \bmod \sum_{\beta>\alpha} C X_{\beta} \\
& \equiv 0 \bmod \sum_{\beta>\alpha} C X_{\beta} .
\end{aligned}
$$

Hence $\operatorname{Ad}\left(n_{1}\right)(H+Z)=H+Z^{\prime}$ where $Z^{\prime} \in \mathfrak{n}_{0} \cap\left(\sum_{\beta>\alpha} C X_{\beta}\right)$. In view of our choice of $Z$ it follows that $H+Z^{\prime}=\operatorname{Ad}\left(n_{2}\right) H$ for some $n_{2} \in N$. Therefore $H+Z=\operatorname{Ad}(n) H$ where $n=n_{1}^{-1} n_{2}$. Since this contradicts our hypothesis the assertion is proved.

Corollary. $A=A_{+} A_{-}$is exactly the centralizer of $A^{0}=A_{+} A_{-}^{0}$ in $G$.

Let $x$ be an element of $G$ which commutes with all elements in $A^{0}$. Then 
it follows from Lemma 7 that $x=u h$ where $u \in A_{-}^{\prime}$ and $h \in A_{+}$. Since $x$ commutes with $A^{0}$ the same is true of $u$. Hence $u$ lies in the centralizer $A_{-}$of $A^{0}$ in $K$.

Since $g_{0}$ is a vector space over $R$ of finite dimension we can regard it as an analytic manifold $\left({ }^{7}\right)$ and identify in the usual way the tangent space at each point $X \in \mathfrak{g}_{0}$ with $\mathfrak{g}_{0}$ itself. Then if $f$ is a function on $\mathfrak{g}_{0}$ which is differentiable at $X$,

$$
Y f(X)=\left\{\frac{d}{d t} f(X+t Y)\right\}_{t=0}
$$

Similar remarks hold also for any linear subspace of $\mathfrak{g}_{0}$ which may also be regarded as an analytic manifold.

Consider the subgroup $A^{0} Z$ in $G$. It is clear that $\mathrm{Ad}\left(A^{0}\right)$ is a maximal connected abelian subgroup of $\operatorname{Ad}(G)$ and therefore it is closed. Hence $A^{0} Z$ is closed in $G$. Let $\varepsilon$ denote the factor space $G / A^{0} Z$ consisting of all cosets of the form $x A^{0} Z(x \in G)$. We regard $\varepsilon$ as an analytic manifold in the usual way (see [1]) and denote by $x \rightarrow \bar{x}$ the natural mapping of $G$ on $\varepsilon$. Then for any fixed $H \in \mathfrak{h}_{0}$, Ad $(x) H$ depends only on $\bar{x}$. We put $\bar{x} H=\operatorname{Ad}(x) H$ $(x \in G)$. It is evident that the mapping $\phi:(\bar{x}, H) \rightarrow \bar{x} H\left(\bar{x} \in \mathcal{E}, H \in \mathfrak{h}_{0}\right)$ is an analytic mapping of $\varepsilon \times \mathfrak{h}_{0}$ into $g_{0}$. We consider the differential ${ }^{(7)}$ of $\phi$. Let $X_{1}, \cdots, X_{r}$ be a base for $\mathfrak{g}_{0} \bmod \mathfrak{h}_{0}$ and let $(d \pi)_{x}$ denote the differential of the natural mapping of $G$ on $\varepsilon_{\text {at }} x$. Then $(d \pi)_{x} X_{i}, 1 \leqq i \leqq r$, forms a base for the tangent space of $\varepsilon$ at $\bar{x}$ (see [1, p. 110]). Hence if we regard the tangent space of $\varepsilon \times \mathfrak{h}_{0}$ at $(\bar{x}, H)$ as the direct sum of the tangent spaces of $\varepsilon$ and $\mathfrak{h}_{0}$ it is easily seen that

$$
\begin{aligned}
d \phi \circ(d \pi)_{x} X_{i} & =-\operatorname{Ad}(x)\left[H, X_{i}\right], & & 1 \leqq i \leqq r, \\
d \phi H_{0} & =\operatorname{Ad}(x) H_{0} & & \left(H_{0} \in \mathfrak{h}_{0}\right) .
\end{aligned}
$$

Let $D$ be the linear mapping of $g_{0}$ into itself defined by $D X_{i}=-\left[H, X_{i}\right]$, $1 \leqq i \leqq r$, and $D H_{0}=H_{0}\left(H_{0} \in \mathfrak{h}_{0}\right)$. Then $D$ defines a linear mapping $\bar{D}$ in the factor space $\mathfrak{g}_{0} / \mathfrak{h}_{0}$ which is the same as that induced by $-\operatorname{ad} H$. Since $G$ is semisimple $\operatorname{det} \operatorname{Ad}(x)=1$. Hence

$$
|\operatorname{det} \operatorname{Ad}(x) D|=|\operatorname{det} D|=|\operatorname{det} \bar{D}|=\prod_{\alpha>0}|\alpha(H)|^{2}
$$

where $\alpha$ runs over all positive roots. This shows that if $H$ is regular $\operatorname{det} \operatorname{Ad}(x) D$ $\neq 0$ and therefore $d \phi$ is regular $\left({ }^{7}\right)$ at $(\bar{x}, H)$. Let $\mathfrak{h}_{1}$ be the set of all regular elements in $\mathfrak{h}_{0}$. Then $\phi$ defines a continuous open mapping of $\varepsilon \times \mathfrak{h}_{1}$ into $\mathfrak{g}_{0}$. The image of $\varepsilon \times \mathfrak{h}_{1}$ in $\mathfrak{g}_{0}$ is obviously the set $\mathfrak{g}_{1}$ of all regular elements in $\mathfrak{g}_{0}$ which are conjugate (under $G$ ) to some element in $\mathfrak{h}_{0}$. Since $\mathfrak{h}_{1}$ is open in $\mathfrak{h}_{0}$, $\mathfrak{g}_{1}$ is open in $\mathfrak{g}_{0}$.

(7) We shall follow the terminology of Chevalley [1] in the rest of this paper. 
Define $A_{-}^{\prime}$ as in Lemma 7 and put $A^{\prime}=A_{-}^{\prime} A_{+}$. Since $\operatorname{Ad}\left(A_{-}^{\prime}\right)$ is the normalizer of $\operatorname{Ad}\left(A^{0}\right)$ in $\operatorname{Ad}(K)$, it is closed and therefore compact. Since $\mathfrak{h}_{\mathfrak{t}_{0}}$ is the normalizer of $\mathfrak{h}_{0}$ in $\mathfrak{f}_{0}$ it follows that $A_{-}^{0}$ is the connected component of $A_{-}^{\prime}$ and therefore $A^{\prime} / A^{0} Z \cong A_{-}^{\prime} / A_{-}^{0} Z \cong \mathrm{Ad}\left(A_{-}^{\prime}\right) / \mathrm{Ad}\left(A_{-}^{0}\right)$ is finite. We denote by $W$ the finite group $A^{\prime} / A^{0} Z$. For any $\bar{x} \in \mathcal{E}$ and $s \in W$ we define $\bar{x} s$ as follows. Choose $x \in G$ and $a \in A^{\prime}$ lying above $\bar{x}$ and $s$ respectively. Then the coset $x a A^{0} Z$ depends only on $\bar{x}$ and $s$ and we define $\bar{x} s$ to be this coset. It is clear that $\bar{x} s \neq \bar{x}$ unless $s=1$. Since $W \subset \mathcal{E}, s H\left(s \in W, H \in \mathfrak{h}_{0}\right)$ is defined and lies in $\mathfrak{h}_{0}$. Now suppose $\bar{x}_{1} H_{1}=\bar{x}_{2} H_{2}\left(\bar{x}_{1}, \bar{x}_{2} \in \mathcal{E} ; H_{1}, H_{2} \in \mathfrak{h}_{1}\right)$. Then if $x_{i}$ lies in $G$ above $\bar{x}_{i}(i=1,2), \operatorname{Ad}\left(x_{1}\right) H_{1}=\operatorname{Ad}\left(x_{2}\right) H_{2}$ and therefore, from Lemma 7 , $x_{1}^{-1} x_{2} \in A^{\prime}$ and $H_{1}=\mathrm{Ad}\left(x_{1}^{-1} x_{2}\right) H_{2}$. Hence there exists an $s \in W$ such that $\bar{x}_{2}=\bar{x}_{1} s$ and $H_{2}=s^{-1} H_{1}$. Moreover $\bar{x}_{2} \neq \bar{x}_{1}$ unless $s=1$. Therefore if $w$ is the order of the group $W$, there are exactly $w$ distinct points in $\varepsilon \times \mathfrak{h}_{1}$ which have the same image in $\mathfrak{g}_{1}$.

Since $A^{0} Z$ is abelian, it is unimodular and therefore (see Weil $[11$, p. 42]) there exists a measure $d \bar{x}$ (which is unique apart from a constant factor) on $\varepsilon$ such that it is invariant under the translations induced on $\varepsilon$ by $G$. Let $n=\operatorname{dim} \mathfrak{g}_{0}$ and let $\left(H_{1}, \cdots, H_{l}\right)$ be a base for $\mathfrak{h}_{0}$ so that $r=n-l$ (in the notation used above). As before let $X_{1}, \cdots, X_{r}$ be a base for $\mathfrak{g}_{0} \bmod \mathfrak{h}_{0}$. Let $\omega$ be a left invariant differential form of degree $n$ on $G$ such that $\omega\left(X_{1}, \cdots, X_{r}\right.$, $\left.H_{1}, \cdots, H_{l}\right)=1$. Then if $\bar{\omega}$ is the differential form of degree $r$ on $\varepsilon$ corresponding to the (suitably normalized) invariant measure we have $\left(^{8}\right)$

$$
\bar{\omega}\left((d \pi)_{x} Y_{1}, \cdots,(d \pi)_{x} Y_{r}\right)=\omega\left(Y_{1}, \cdots, Y_{r}, H_{1}, \cdots, H_{l}\right)
$$

for any $Y_{1}, \cdots, Y_{r} \in g_{0}$. Let $d X$ denote the Euclidean measure on $g_{0}$ and $\eta$ the corresponding differential form of degree $n$ on $\mathfrak{g}_{0}$. We may assume that $\eta\left(X_{1}, \cdots, X_{r}, H_{1}, \cdots, H_{l}\right)=1$. Consider the image of $\eta$ under the dual $\left(^{7}\right)$ $\delta \phi$ of the mapping $d \phi$. Then $(\delta \phi \eta)_{\bar{x}, H}$ is the form defined at $(\bar{x}, H)$ on $\varepsilon \times \mathfrak{h}_{0}$ by the rule

$$
\begin{aligned}
(\delta \phi \eta)_{\bar{x}, H}\left((d \pi)_{x} X_{1},\right. & \left.\cdots,(d \pi)_{x} X_{r}, H_{1}, \cdots, H_{l}\right) \\
& =\eta_{\bar{x} H}\left(d \phi \circ(d \pi)_{x} X_{1}, \cdots, d \phi \circ(d \pi)_{x} X_{r},(d \phi) H_{1}, \cdots,(d \phi) H_{l}\right) \\
& = \pm \prod_{\alpha>0}|\alpha(H)|^{2}
\end{aligned}
$$

as we saw above. Let $\xi$ be the differential form on $\mathfrak{h}_{0}$ corresponding to the Euclidean measure $d H$. We assume that $\xi\left(H_{1}, \cdots, H_{l}\right)=1$. Then

$$
\begin{aligned}
(\delta \phi \eta)_{\tilde{x} H}\left((d \pi)_{x} X_{1}, \cdots,\right. & \left.(d \pi)_{x} X_{r}, H_{1}, \cdots, H_{l}\right) \\
& = \pm \prod_{\alpha>0}|\alpha(H)|^{2} \bar{\omega}\left((d \pi)_{x} X_{1}, \cdots,(d \pi)_{x} X_{r}\right) \xi\left(H_{1}, \cdots, H_{l}\right)
\end{aligned}
$$

(8) We give here once in some detail the computation of the measure on $g_{1}$ in terms of the measure on $\mathcal{E}$ and $\mathfrak{h}_{1}$. All subsequent computations of a similar sort will be sketched only very briefly. 
This proves that

$$
(\delta \phi \eta)_{\bar{x}, H}= \pm \prod_{\alpha>0}|\alpha(H)|^{2} \zeta
$$

where $\zeta$ is the differential form on $\varepsilon \times \mathfrak{h}_{0}$ corresponding to the product measure $d \bar{x} d H$. Therefore taking into account the fact that every point in $\mathrm{g}_{1}$ has exactly $w$ distinct pre-images in $\mathcal{E} \times \mathfrak{h}_{1}$, we can conclude that

$$
w \int_{\mathfrak{g}_{1}} f(X) d X=\int \varepsilon_{\times_{\mathfrak{h}_{1}}} \prod_{\alpha>0}|\alpha(H)|^{2} f(\bar{x} H) d \bar{x} d H
$$

for any measurable function $f$ on $g_{1}$, whenever at least one of the two sides of this equation remains finite on replacing $f$ by $|f|$.

Now let $G_{*}=G / Z$ and let $x \rightarrow x_{*}$ denote the natural mapping of $G$ on $G_{*}$. Since $Z \subset K, A_{+}$and $N$ are mapped isomorphically and so we may identify them with their images under this mapping. Then $G_{*}=K_{*} A_{+} N$. Now an $=a n a^{-1} \cdot a\left(a \in A_{+}, n \in N\right)$. Hence $G_{*}=K_{*} N A_{+}$and $K_{*} N \cap A_{+}=\{1\}$. Therefore $G_{*} / A_{+}$is homeomorphic to $K_{*} N$ under the mapping $u_{*} n \rightarrow u_{*} n A_{+}$ $\left(u_{*} \in K_{*}, n \in N\right)$. If we identify $G_{*} / A_{+}$with $K_{*} N$ under this mapping it is easy to verify that $d u_{*} d n$ is the invariant measure on $G_{*} / A_{+}$. (Here $d u_{*}$ and $d n$ are the Haar measures on $K_{*}$ and $N$ respectively.) Now $\varepsilon=G / A^{0} Z$ $=G_{*} / A_{*}^{0}$ where $A_{*}^{0}=A_{+} A_{-*}^{0}$ and $A_{-*}^{0}$ is the analytic subgroup of $K_{*}$ corresponding to $\mathfrak{h}_{\mathfrak{t}_{0}}$. Let $d x_{*}, d h$, and $d h_{*}$ be the Haar measures on $G_{*}, A_{+}$, and $A_{-*}^{0}$ respectively. Then $d x_{*}=d u_{*} d n d h\left(x_{*}=u_{*} n h\right)$ if $d n$ is suitably normalized. We shall assume that $\int d h_{*}=1$. Let $d \bar{x}$ be the invariant measure on $\varepsilon$. Then if $d \bar{x}$ is suitably normalized, $d x_{*}=d \bar{x} d h_{*} d h$ in the sense of $[11, \mathrm{p} .42]$. Let $F(\bar{x})$ be a continuous function on $\varepsilon$ which vanishes outside a compact set. Then there exists (see $[11$, p. 43$]$ ) a continuous function $F_{1}$ on $G_{*}$ vanishing outside a compact set such that

$$
F\left(\bar{x}_{*}\right)=\int F_{1}\left(x_{*} h h_{*}\right) d h_{*} d h
$$

where $x_{*} \rightarrow \bar{x}_{*}$ is the natural mapping of $G_{*}$ on $\varepsilon=G_{*} / A_{*}$. Put

$$
F_{2}\left(x_{*}\right)=\int F_{1}\left(x_{*} h_{*}\right) d h^{*}
$$

Then

$$
\int F_{2}\left(x_{*}\right) d x_{*}=\int F(\hat{x}) d \bar{x}
$$

But

$$
\int F_{2}\left(x_{*}\right) d x_{*}=\int F_{2}\left(u_{*} n h\right) d u_{*} d n d h=\int F\left(\overline{u_{*} n}\right) d u_{*} d n
$$


since

$$
\int F_{2}\left(u_{*} n h\right) d h=\int F_{1}\left(u_{*} n h h_{*}\right) d h d h_{*}=F\left(\overline{u_{*} n}\right)
$$

Therefore

$$
\int F(\bar{x}) d \bar{x}=\int F\left(\overline{u_{*} n}\right) d u_{*} d n
$$

and from this the same relation follows for any measurable function $F$ on $\varepsilon$ provided either one of the two sides of the above equation remains finite on replacing $F$ by $|F|$. Therefore in particular

$$
\int_{\varepsilon} f(\bar{x} H) d \bar{x}=\int_{K \cdot N} f\left(\operatorname{Ad}\left(u_{*} n\right) H\right) d u_{*} d n
$$

where $x_{*} \rightarrow \operatorname{Ad}\left(x_{*}\right)$ is the adjoint representation of $G_{*}$. Hence

$$
w \int_{\mathfrak{g}_{1}} f(X) d X=\int_{K_{*}} d u_{*} \int_{N \times \mathfrak{b}_{1}} f\left(\operatorname{Ad}\left(u_{*} n\right) H\right) d n d H .
$$

We have seen (Lemma 8$)$ that the mapping $(n, H) \rightarrow \operatorname{Ad}(n) H$ is a 1-1 mapping of $N \times \mathfrak{h}_{1}$ onto $\mathfrak{h}_{1}+\mathfrak{n}_{0}$ which is obviously analytic. Now

$$
\begin{array}{ll}
\lim _{t \rightarrow 0} \frac{1}{t}[\operatorname{Ad}(n \exp t X) H-\operatorname{Ad}(n) H]=-\operatorname{Ad}(n)[H, X] & \left(X \in \mathfrak{n}_{0}\right), \\
\lim _{t \rightarrow 0} \frac{1}{t}\left[\operatorname{Ad}(n)\left(H+t H_{0}\right)-\operatorname{Ad}(n) H\right]=\operatorname{Ad}(n) H_{0} \quad\left(H_{0} \in \mathfrak{h}_{0}\right) .
\end{array}
$$

Hence if $D$ is the linear mapping of $\mathfrak{h}_{0}+\mathfrak{n}_{0}$ into itself given by $D X=-(\operatorname{ad} H) X$ $\left(X \in \mathfrak{n}_{0}\right)$ and $D H_{0}=H_{0}\left(H_{0} \in \mathfrak{h}_{0}\right)$ it follows that

$$
|\operatorname{det}(\operatorname{Ad}(n) D)|=|\operatorname{det} D|=\prod_{\alpha \in P+}|\alpha(H)|
$$

as the determinant of the restriction of Ad $(n)$ on $\mathfrak{h}_{0}+\mathfrak{n}_{0}$ is obviously 1 . Since $H \in \mathfrak{h}_{1}, \prod_{\alpha \in P_{+}}|\alpha(H)| \neq 0$ and our mapping is regular at $(n, H)$. Therefore in view of Lemma 8 , it defines a topological mapping of $\mathfrak{h}_{1} \times N$ onto $\mathfrak{h}_{1}+\mathfrak{n}_{0}$ and $\int_{N \times \mathfrak{G}_{1}} f\left(\operatorname{Ad}\left(u_{*} n\right) H\right) d n d H=\int_{\mathfrak{h}_{1}+\mathfrak{n}_{0}} \coprod_{\alpha \in P_{+}}|\alpha(H)|^{-1} f\left(\operatorname{Ad}\left(u_{*}\right)(H+Z)\right) d H d Z$ where $d Z$ is the suitably normalised Euclidean measure on $\mathfrak{n}_{0}$. Therefore

$$
\begin{aligned}
& w \int_{\mathfrak{g}_{1}} f(X) d X \\
& \quad=\int_{\mathfrak{b}_{1}+\mathfrak{n}_{0}} \prod_{\alpha \in P_{+}}|\alpha(H)| \prod_{\alpha \in P_{-}}|\alpha(H)|^{2} d H d Z \int_{K_{*}} f\left(\operatorname{Ad}\left(u_{*}\right)(H+Z)\right) d u_{*} .
\end{aligned}
$$


Thus we have proved the following result.

Lemma 9. It is possible to normalise the Euclidean measures $d X, d H$, and $d Z$ on $\mathfrak{g}_{0}, \mathfrak{h}_{0}$, and $\mathfrak{n}_{0}$ respectively in such a way that for any measurable function $f(X)$ on $\mathfrak{g}_{1}$,

$$
\begin{aligned}
& \int_{\mathfrak{g}_{1}} f(X) d X \\
&=\int_{\mathfrak{g}_{1}+\mathfrak{n}_{0}} \prod_{\alpha \in P_{+}}|\alpha(H)| \prod_{\alpha \in P_{-}}|\alpha(H)|^{2} d H d Z \int_{K_{0}} f\left(\operatorname{Ad}\left(u_{*}\right)(H+Z)\right) d u_{*}
\end{aligned}
$$

provided at least one of these two integrals remains finite on replacing $f$ by $|f|$.

We shall call an element $x \in G$ singular if the characteristic polynomial of $\operatorname{Ad}(x)$ in the indeterminate $\lambda$ is divisible by $(1-\lambda)^{l+1}\left(l=\operatorname{dim} \mathfrak{h}_{0}\right)$. If $x$ is not singular we say it is regular. Let $H \in \mathfrak{h}_{0}$. Then $h=\exp H$ is singular if and only if $\alpha(H)=2 \pi m(-1)^{1 / 2}$ for some root $\alpha$ and some integer $m$. Let $n \in N$. Then it is easily seen that Ad $(h)$, Ad $(h n)$ have the same characteristic polynomials. Hence $h n$ is singular if and only if $h$ is singular.

Lemma 10. Let $H$ be an element in $\mathfrak{h}_{0}$ such that $h=\exp H$ is regular. Then $X \rightarrow h^{-1} \exp (H+X)\left(X \in \mathfrak{n}_{0}\right)$ is a 1-1 analytic mapping of $\mathfrak{n}_{0}$ onto $N$ which is everywhere regular.

We denote by $\left(1-e^{-\lambda}\right) / \lambda$ the power series $\sum_{m \geqq 0}(-1)^{m} \lambda^{m} /(m+1)$ ! which is convergent for all values of $\lambda$. If $B$ is a matrix or an endomorphism of a finite-dimensional (real or complex) vector space we put

$$
\frac{1-e^{-B}}{B}=\sum_{m \geq 0}(-1)^{m} \frac{B^{m}}{(m+1) !} .
$$

In the proof of the above lemma we shall make use of the following known result (see Chevalley [1, p. 157]). If $Y \in \mathfrak{g}_{0}$ and $f$ is a function on $G$ differentiable at $y=\exp Y$, then

$$
\left\{\frac{d}{d t} f(\exp (Y+t Z))\right\}_{t=0}=\left(Z^{\prime} f\right)(y) \quad\left(Z \in g_{0}\right)
$$

where

$$
Z^{\prime}=\left(\frac{1-e^{-\mathbf{a d} Y}}{\operatorname{ad} Y}\right) Z .
$$

Now let $\phi(X)=h^{-1} \exp (H+X)\left(X \in \mathfrak{n}_{0}\right)$. Then

$$
(d \phi)_{X} Y=\frac{1-\exp \{-\operatorname{ad}(H+X)\}}{\operatorname{ad}(H+X)} Y \quad\left(Y \in \mathfrak{n}_{0}\right) .
$$


Clearly $\mathfrak{n}_{0}$ is invariant under ad $(H+X)$. Let $D$ denote the restriction of ad $(H+X)$ on $\mathfrak{n}_{0}$. We extend $D$ on $\mathfrak{n}$ by linearity. Then the matrix of $D$ with respect to the base $X_{\alpha}\left(\alpha \in P_{+}\right)$is in triangular form and therefore it is clear that

$$
\operatorname{det}\left(\frac{1-e^{-D}}{D}\right)=\coprod_{\alpha \in P_{+}} \frac{1-e^{-\alpha(H)}}{\alpha(H)} \neq 0
$$

since $h$ is regular. This proves that $\phi$ is everywhere regular.

We claim moreover that $h^{-1} \exp (H+X) \neq 1 \quad\left(X \in \mathfrak{n}_{0}\right)$ unless $X=0$. For suppose $X \neq 0$. Then $X=a_{\alpha} X_{\alpha}+\sum_{\beta>\alpha} a_{\beta} X_{\beta}$ where $\alpha \in P_{+}, a_{\alpha}, a_{\beta} \in C$, and $a_{\alpha} \neq 0$. Hence for any $H_{1} \in \mathfrak{h}_{0}$,

$$
\begin{aligned}
\operatorname{Ad}(\exp (H+X)) H_{1} & =\exp (\operatorname{ad}(H+X)) H_{1} \\
& \equiv H_{1}-a_{\alpha} \alpha\left(H_{1}\right) X_{\alpha} \bmod \sum_{\beta>\alpha} C X_{\beta} .
\end{aligned}
$$

Therefore if $\alpha\left(H_{1}\right) \neq 0, \operatorname{Ad}(\exp (H+X)) H_{1} \neq H_{1}$. This proves that $\exp (H+X)$ $\neq h$.

Now suppose $\phi\left(X_{1}\right)=\phi\left(X_{2}\right) \quad\left(X_{1}, \quad X_{2} \in \mathfrak{n}_{0}\right)$. Then $\exp \quad\left(H+X_{1}\right)$ $=\exp \left(H+X_{2}\right)$. Since $H$ is regular in $\mathfrak{h}_{0}$, it follows from Lemma 8 that $H+X_{i}=\operatorname{Ad}\left(n_{i}\right) H\left(n_{i} \in N, i=1,2\right)$. Hence if $n_{1}^{-1} n_{2}=n$ and $\operatorname{Ad}(n) H=H+X$ $\left(X \in \mathfrak{n}_{0}\right)$, we get $\exp (H+X)=h$. Therefore in view of the above result $X=0$ and so from Lemma $8, n=1$. This proves that $n_{1}=n_{2}$ and $X_{1}=X_{2}$. Therefore $\phi$ is univalent.

Let $V=\phi\left(\mathfrak{n}_{0}\right)$. Since $\phi$ is everywhere regular, $V$ is an open subset of $N$. In order to prove the lemma it only remains to show that $V=N$. Let $n \in N$. Since $N$ is nilpotent, $n=\exp X$ for some $X \in \mathfrak{n}_{0}$. Consider the one-parameter group $\exp t X(t \in R)$. Let $T$ be the subset of $R$ consisting of all $t$ such that $\exp t X \in V$. Since $V$ is open, $T$ is an open set containing zero. Let $T_{0}$ be the connected component of zero in $T$. Put $Z(t)=\phi^{-1}(\exp t X)\left(t \in T_{0}\right)$. Since $\phi$ defines an analytic isomorphism of $\mathfrak{n}_{0}$ with $V, t \rightarrow Z(t)$ is an analytic mapping of $T_{0}$ into $\mathfrak{n}_{0}$. Moreover $\exp (H+Z(t))=\exp H \exp t X\left(t \in T_{0}\right)$. From this it follows immediately that

$$
\frac{1-\exp (-\operatorname{ad}(H+Z(t)))}{\operatorname{ad}(H+Z(t))} \dot{Z}(t)=X
$$

where $\dot{Z}(t)=\lim _{\epsilon \rightarrow 0}(1 / \epsilon)(Z(t+\epsilon)-Z(t))(\epsilon \in R)$. Hence

$$
(1-\exp (-\operatorname{ad}(H+Z(t))) \dot{Z}(t)=\operatorname{ad}(H+Z(t)) X .
$$

Let $\theta$ be the automorphism of $g_{0}$ such that $\theta\left(Y_{1}+Y_{2}\right)=Y_{1}-Y_{2} \quad\left(Y_{1} \in \mathfrak{l}_{0}\right.$, $\left.Y_{2} \in p_{0}\right)$. Then (see $[6, \S 2]$ )

$$
Q(Y)=-\operatorname{sp}(\operatorname{ad}(\theta(Y)) \text { ad } Y)
$$

is a positive definite quadratic form on $g_{0}$. Put $|Y|^{2}=Q(Y)$ and let $D(t)$ de- 
note the restriction of $1-\operatorname{Ad}\left((\exp H \exp t X)^{-1}\right)$ on $\mathfrak{n}_{0}$. Then $\operatorname{det} D(t)$ $=\prod_{\alpha \in P_{+}}\left(1-e^{-\alpha(H)}\right) \neq 0$. Hence $D(t)$ is nonsingular and

$$
\dot{Z}(t)=D(t)^{-1} \text { ad }(H+Z(t)) X .
$$

For any endomorphism $A$ of $\mathfrak{n}_{0}$ let $\|A\|^{2}$ denote the sum of the squares of the matrix coefficients of $A$ relative to any base of $\mathfrak{n}_{0}$ which is orthonormal with respect to the quadratic form $Q$. Then it is clear that

$$
|\dot{Z}(t)| \leqq\left\|D(t)^{-1}\right\| \mid \text { ad }(H+Z(t)) X \mid
$$

and

$$
|\operatorname{ad}(H+Z(t)) X| \leqq|[H, X]|+|[Z(t), X]| \leqq p+q|Z(t)|
$$

where $p$ and $q$ are some positive numbers independent of $t$. Now it is evident that $D(t)$, and therefore $D(t)^{-1}$, depends continuously on $t$. Hence $\left\|D(t)^{-1}\right\|$ is bounded on every bounded subset of $R$. Therefore given any positive number $t_{0}$, there exists a constant $M$ such that $\left\|D(t)^{-1}\right\| \leqq M$ if $|t| \leqq t_{0}$. Now suppose $t \in T_{0}$ and $|t| \leqq t_{0}$. Then, if we denote the corresponding bilinear form also by $Q$,

$$
\frac{1}{2} \frac{d}{d t}|Z(t)|^{2}=Q(Z(t), \dot{Z}(t)) \leqq|Z(t)||\dot{Z}(t)| .
$$

Hence, if $t \neq 0$,

$$
\frac{d}{d t}|Z(t)| \leqq|\dot{Z}(t)| \leqq M(p+q|Z(t)|) .
$$

From this it follows by integration that

$$
1+\frac{q}{p}|Z(t)| \leqq e^{M q|t|}
$$

provided $|t| \leqq t_{0}$ and $t \in T_{0}$. This proves that $|Z(t)|$ remains bounded so long as $t$ remains bounded in $T_{0}$.

We shall now show that $T_{0}$ is closed in $R$. Let $t_{k}$ be a sequence in $T_{0}$ which converges to $t \in R$. Then $t_{k}$ remains bounded and therefore $\left|Z\left(t_{k}\right)\right|$ also remains bounded. Since every bounded closed subset of $\mathfrak{n}_{0}$ is compact, we can choose a subsequence $t_{k_{i}}$ such that $Z\left(t_{k_{i}}\right)$ converges to a limit $Z$ in $\mathfrak{n}_{0}$. Then

$$
\phi(Z)=\lim _{i \rightarrow \infty} \phi\left(Z\left(t_{k_{i}}\right)\right)=\lim _{i \rightarrow \infty} \exp t_{k_{i}} X=\exp t X .
$$

This proves that $t \in T$. But $T_{0}$, being a component of $T$, is closed in $T$. Therefore $t \in T_{0}$. Hence $T_{0}$ is both open and closed in $R$. Since $R$ is connected and $0 \in T_{0}, T_{0}=R$. Therefore $n=\exp X \in V$. This proves that $V=N$. 
COROLlARy. Let $h$ be a regular element in $A^{0}$. Then the mapping $n \rightarrow h^{-1} n h n^{-1}$ $(n \in N)$ is a topological mapping of $N$ onto itself.

Choose $H \in \mathfrak{h}_{0}$ such that $h=\exp H$. Then $n h n^{-1}=\exp (\operatorname{Ad}(n) H)$ $=\exp (H+X(n))$ where $X(n)=\operatorname{Ad}(n) H-H \in \mathfrak{n}_{0}$. But if $Y \in \mathfrak{n}_{0}$,

$$
\lim _{t \rightarrow 0} \frac{1}{t}\{X(n \exp t Y)-X(n)\}=-\operatorname{Ad}(n)[H, Y] \text {. }
$$

Therefore if $D$ is the restriction of adH on $\mathfrak{n}_{0}$,

$$
|\operatorname{det} \operatorname{Ad}(n) D|=|\operatorname{det} D|=\coprod_{\alpha \in P_{+}}|\alpha(H)| \neq 0
$$

since $H$ is regular. Therefore the mapping $n \rightarrow X(n)$ is everywhere regular. Hence it is a topological mapping of $N$ onto $\mathfrak{n}_{0}$ from Lemma 8 . The corollary now follows immediately from Lemma 10.

We shall also prove the following lemma which will be useful later.

LemMa 11. There exists a neighbourhood $U$ of zero in $\mathfrak{h}_{0}$ such that the exponential mapping is univalent and regular on $U+\mathfrak{n}_{0}$ and $\exp H$ is regular in $G$ for every $H \neq 0$ in $U$.

It is obvious that there exists a neighbourhood $U$ of zero in $\mathfrak{h}_{0}$ such that $\exp H$ is regular for all $H \neq 0$ in $U$ and the mapping $H \rightarrow \exp H$ is univalent on $U$. Now we know (see Chevalley [1, p. 157]) that the exponential mapping is regular at a point $X \in g_{0}$ if and only if $\operatorname{det}((1-\exp (-\operatorname{ad} X)) / \operatorname{ad} X) \neq 0$. But if $H \in U$ and $X \in \mathfrak{n}_{0}, \quad \operatorname{det}((1-\exp (-\operatorname{ad}(H+X))) / \operatorname{ad}(H+X))$ $=\operatorname{det}\left(\left(1-e^{-\mathrm{ad} H}\right) / \operatorname{ad} H\right)=\prod_{\alpha>0}\left(\left(1-e^{-\alpha(H)}\right) / \alpha(H)\right) \prod_{\alpha>0}\left(e^{\alpha(H)}-1\right) / \alpha(H) \neq 0$. Hence the exponential mapping is regular on $U+\mathfrak{n}_{0}$. Now suppose $\exp \left(H_{1}+X_{1}\right)$ $=\exp \left(H_{2}+X_{2}\right)\left(H_{1}, H_{2} \in U ; X_{1}, X_{2} \in \mathfrak{n}_{0}\right)$. Let $h_{i}=\exp H_{i}(i=1,2)$. Then it is clear that $h_{2} \in h_{1} N$ and therefore $h_{1}^{-1} h_{2} \in N \cap A^{0}=\{1\}$. Since the exponential mapping is univalent on $U$ it follows that $H_{1}=H_{2}$. Put $H=H_{1}=H_{2}$. Then if $H=0, \exp X_{1}=\exp X_{2}$. Since the exponential mapping is well known to be univalent on $\mathfrak{n}_{0}, X_{1}=X_{2}$. On the other hand suppose $H \neq 0$. Then exp $H$ is regular and therefore from Lemma $10, X_{1}=X_{2}$. This proves the lemma.

Corollary. The exponential mapping maps $U+\mathfrak{n}_{0}$ topologically into $G$. Moreover if $U$ is compact, $\exp \left(U+\mathfrak{n}_{0}\right)$ is closed in $G$.

The first assertion is obvious from Lemma 11. Moreover it follows from Lemma 10 that $\exp \left(U+\mathfrak{n}_{0}\right)=(\exp U) N$. Therefore if $U$ is compact, exp $U$ is also compact and therefore $(\exp U) N$ is closed.

Let $G_{1}$ be the set of all regular elements of $G$ which are conjugate to some element in $A^{0} Z$. Let $A_{1}$ be the set of all regular elements in $A^{0}$. Define the factor space $\mathcal{E}=G / A^{0} Z$ as before. For any $\bar{x} \in \mathcal{E}$ and $h \in A^{0} Z$ define $h^{\bar{x}}=x h x^{-1}$ where $x$ is any element of the $\operatorname{coset} \bar{x}$. Then $\phi:(\bar{x}, h) \rightarrow h^{\bar{x}}$ is a continuous mapping of $\varepsilon \times A^{0} Z$ into $G$ and $\phi\left(\varepsilon \times A_{1} Z\right)=G_{1}$. We shall prove that $\phi$ is regular 
on $\mathcal{E} \times A_{1} Z$. Since $A_{1} Z$ is obviously open in $A^{0} Z$, it would follow that $G_{1}$ is open in $G$. Let $X_{1}, \cdots, X_{r}$ be a base for $\mathfrak{g}_{0} \bmod \mathfrak{h}_{0}$ and let $\pi$ denote the natural mapping of $G$ on $\mathcal{E}$. Then if $x \in \bar{x}$ we know that $(d \pi)_{x} X_{i}, 1 \leqq i \leqq r$, is a base for the tangent space of $\varepsilon$ at $\bar{x}$. Now if $X \in \mathfrak{g}_{0}$ and $H \in \mathfrak{h}_{0}$,

$$
x \exp t X h(x \exp t X)^{-1}=x h x^{-1} \exp t \operatorname{Ad}\left(x h^{-1}\right) X \exp (-t \operatorname{Ad}(x) X),
$$

and

$$
x h \exp t H x^{-1}=x h x^{-1} \exp t \operatorname{Ad}(x) H .
$$

Therefore

$$
\begin{aligned}
d \phi \circ(d \pi)_{x} X_{i} & =\operatorname{Ad}(x)\left[\operatorname{Ad}\left(h^{-1}\right)-1\right] X_{i}, \\
d \phi H & =\operatorname{Ad}(x) H .
\end{aligned}
$$

Then if $D$ is the endomorphism of $\mathfrak{g}_{0}$ such that

$$
\begin{aligned}
D X_{i} & =\left(\operatorname{Ad}\left(h^{-1}\right)-1\right) X_{i}, & 1 \leqq i \leqq r, \\
D H & =H & \left(H \in \mathfrak{h}_{0}\right),
\end{aligned}
$$

it is clear that

$$
|\operatorname{det} \operatorname{Ad}(x) D|=|\operatorname{det} D|=\left|\prod_{\alpha>0}\left(e^{-\alpha(H)}-1\right) \prod_{\alpha>0}\left(e^{\alpha(H)}-1\right)\right|
$$

where $H$ is any element in $\mathfrak{h}_{0}$ such that $h^{-1} \exp H \in Z$. Put

$$
\Delta(h)=\left|\prod_{\alpha>0}\left(e^{\alpha(\boldsymbol{H}) / 2}-e^{-\alpha(\boldsymbol{H}) / 2}\right)\right| .
$$

Then if $h$ is regular, $\mid \operatorname{det}\left(\operatorname{Ad}(x) D \mid=\Delta^{2}(h) \neq 0\right.$ and this shows that $\phi$ is regular on $\varepsilon \times A_{1} Z$. Now suppose $x$ and $h$ are two elements in $G$ and $A_{1} Z$ respectively such that $x h x^{-1} \in A^{0} Z$. The set of all points in $\mathfrak{g}_{0}$ which are left fixed by Ad $(h)$ is exactly $\mathfrak{h}_{0}$. Hence the corresponding set of fixed points for Ad $\left(x h x^{-1}\right)$ is Ad $(x) \mathfrak{h}_{0}$. But since $x h x^{-1} \in A^{0} Z$ it follows that $\mathfrak{h}_{0} \subset A d(x) \mathfrak{h}_{0}$. Therefore $\mathfrak{h}_{0}=\operatorname{Ad}(x) \mathfrak{h}_{0}$ (because $\operatorname{dim} \operatorname{Ad}(x) \mathfrak{h}_{0}=\operatorname{dim} \mathfrak{h}_{0}$ ) and $x \in A^{\prime}$ (Lemma $7)$. From this we deduce easily that the complete inverse image under $\phi$ of any point in $G_{1}$ consists of exactly $w$ distinct points ( $w$ is the order of $W$ $=A^{\prime} / A^{0} Z$ ). Therefore the method used in the proof of Lemma 9 permits us to conclude that

$$
\int_{G_{1}} f(x) d x=\int_{K_{*}} d u_{*} \int_{N \times A_{1} Z} f\left(\left(n h n^{-1}\right)^{u_{*}}\right) \Delta^{2}(h) d n d h
$$

for any measurable function $f$ on $G_{1}$ for which at least one of the two integrals above remains finite on replacing $f$ by $|f|$. Here $d x, d n$, $d h$ are the suitably normalised Haar measures on $G, N$, and $A^{0} Z$ respectively. 

Then

Now consider the mapping $\psi:(h, n) \rightarrow n h n^{-1}$ of $A^{0} Z \times N$ into $A^{0} Z N$.

$$
n h \exp t H n^{-1}=n h n^{-1} \exp t \operatorname{Ad}(n) H \quad\left(H \in \mathfrak{h}_{0}\right),
$$

$(n \exp t X) h(n \exp t X)^{-1}$

$$
=n h n^{-1} \exp t \operatorname{Ad}\left(n h^{-1}\right) X \exp (-t \operatorname{Ad}(n) X) \quad\left(X \in \mathfrak{n}_{0}\right) .
$$

This shows that

$$
\begin{aligned}
& (d \psi) H=\operatorname{Ad}(n) H \\
& \left(H \in \mathfrak{h}_{0}\right) \text {, } \\
& (d \psi) X=\operatorname{Ad}(n)\left[\operatorname{Ad}\left(h^{-1}\right)-1\right] X \\
& \left(X \in \mathfrak{n}_{0}\right) \text {. }
\end{aligned}
$$

Hence

$$
|\operatorname{det}(d \psi)|=\left|\prod_{\alpha \in P_{+}}\left(e^{-\alpha(H)}-1\right)\right|=\left|e^{-\rho_{+}(H)}\right|\left|\prod_{\alpha \in P_{+}}\left(e^{\alpha(H) / 2}-e^{-\alpha(H) / 2}\right)\right|
$$

where $\rho_{+}=2^{-1} \sum_{\alpha \in P_{+}} \alpha$ and $H$ is any element in $\mathfrak{h}_{0}$ such that $h^{-1} \exp H \in Z$. Suppose $H=H_{+}+H_{-}$where $H_{+} \in \mathfrak{h}_{\mathfrak{p}_{0}}$ and $H_{-} \in \mathfrak{h}_{\mathfrak{p}_{0}}$. Then $\rho_{+}\left(H_{-}\right)$is purely imaginary while $\rho_{+}\left(H_{+}\right)$is real and equal to $\rho\left(H_{+}\right)$. Hence

$$
|\operatorname{det}(d \psi)|=e^{-\rho(H+)}\left|\prod_{\alpha \in P_{+}}\left(e^{\alpha(\boldsymbol{H}) / 2}-e^{-\alpha(\boldsymbol{H}) / 2}\right)\right| .
$$

Since $Z \cap A_{+}=\{1\}, H_{+}$is uniquely determined by $h$. Therefore if we put

$$
\Delta_{+}(h)=\left|\prod_{\alpha \in P_{+}}\left(e^{\alpha(H) / 2}-e^{-\alpha(H) / 2}\right)\right|,
$$

$\Delta_{+}$is a well-defined function on $A^{0} Z$. Now if we take into account the fact that the Haar measure on $A^{0} Z N$ is $d s=d h d n\left(s=h n ; h \in A^{0} Z, n \in N\right)$ we find from the corollary to Lemma 11 that

$$
\int_{N \times A_{1} Z} f\left(n h n^{-1}\right) \exp \left\{-\rho\left(\log h_{+}\right)\right\} \Delta_{+}(h) d h d n=\int_{A_{1} Z_{N}} f(h n) d h d n .
$$

Here $h_{+}$denotes the unique element in $A_{+}$such that $h^{-1} h_{+} \in A_{-}^{0} Z$. Comparing this with our earlier result we get

$$
\int_{G_{1}} f(x) d x=\int_{K_{*}} d u_{*} \int_{A_{1} Z N} f\left((h n) u_{*}^{u_{*}} e^{\rho(\log h+)} \Delta_{+}(h) \Delta(h)^{2} d h d n .\right.
$$

Let $\Lambda$ be a linear function on $\mathfrak{h}_{\mathfrak{p}_{0}}$ and let $\delta \in \omega_{M_{0} Z}$ (see $\S 3$ for notation). Put

$$
\gamma_{1}(h)=\left\{\Delta_{+}(h)\right\}^{-1} \exp \left\{(\Lambda+\rho)\left(\log h_{+}\right)\right\} \xi_{\delta}\left(h_{-}\right) \quad\left(h \in A_{1} Z\right) .
$$

Here $h_{+}$and $h_{-}$are the unique elements in $A_{+}$and $A_{-}^{0} Z$ respectively such that $h=h_{+} h_{-}$. Moreover let 


$$
\gamma(h)=\sum_{s \in W} \gamma_{1}\left(h^{s}\right)
$$

and consider the mapping $(\bar{x}, h) \rightarrow h^{\bar{x}}$ of $\varepsilon \times A_{1} Z$ onto $G_{1}$. It is everywhere open and continuous and the complete inverse image of $h^{\bar{x}}$ under this mapping is the set $\left(\bar{x} s^{-1}, h^{s}\right)(s \in W)$. Therefore if we put

$$
\Theta_{\Lambda, \delta}\left(h^{\bar{x}}\right)=\gamma(h) \quad\left(\bar{x} \in \mathcal{E}, h \in A_{1} Z\right)
$$

we clearly get a continuous function on $G_{1}$. Then

$$
\begin{aligned}
\int_{G_{1}} \mid & f(x) \Theta_{\Delta, \delta}(x) \mid d x \\
& =\int_{\mathcal{E}_{\times_{A_{1} Z}}}\left|f\left(h^{\bar{x}}\right) \gamma(h)\right| \Delta^{2}(h) d \bar{x} d h \\
& \leqq w \int_{K_{*}} d u_{*} \int_{N \times_{A_{1} Z}}\left|f\left(\left(n h n^{-1}\right)^{u_{*}}\right) \gamma_{1}(h)\right| \Delta^{2}(h) d \bar{x} d h \\
& =w \int_{K_{*}} d u_{*} \int_{A_{1} Z N}\left|f\left((h n)^{u^{*}}\right) \gamma_{1}(h)\right| \exp \left\{\rho\left(\log h_{+}\right)\right\} \Delta_{+}(h) \Delta_{-}^{2}(h) d n d h \\
& =w \int_{K_{*}} d u_{*} \int_{A_{1} Z N}\left|f\left((h n)^{u_{*}}\right) \exp \left\{(\Lambda+2 \rho)\left(\log h_{+}\right)\right\} \xi_{\delta}\left(h_{-}\right)\right| \Delta_{-}^{2}(h) d n d h
\end{aligned}
$$

where $\Delta_{-}(h)=\Delta(h) / \Delta_{+}(h)=\left|\prod_{\alpha \in P_{-}}\left(e^{\alpha(H) / 2}-e^{-\alpha(H) / 2}\right)\right|, H$ being any element in $\mathfrak{h}_{0}$ such that $h^{-1} \exp H \in Z$. Now suppose $f$ is a continuous function on $G$ which vanishes outside a compact set. Then the right-hand side is clearly finite. Hence $f(x) \Theta_{\Lambda, \delta}(x)$ is integrable on $G_{1}$. Hence we may apply the above argument to $f(x) \Theta_{\Lambda, \delta}(x)$ instead of $\left|f(x) \Theta_{\Lambda, \delta}(x)\right|$ and conclude from Lemma 6 that

$$
\int_{G_{1}} f(x) \Theta_{\Lambda, \delta}(x) d x=c T_{\Lambda, \delta}(f)
$$

since $\Delta_{-}(h)=\Delta_{-}\left(h_{-}\right)$. Here $c$ is a positive constant which is independent of $\Lambda, \delta$, or $f$.

Let $s$ be any element in $W$. Then there exists an element $u \in K$ such that Ad $(u) H=s H$ for all $H \in \mathfrak{h}_{0}$. Since Ad $(u)$ leaves $\mathfrak{p}_{0}$ and $\mathfrak{l}_{0}$ invariant it follows that $\operatorname{Ad}(u) \mathfrak{h}_{\mathfrak{p}_{0}} \subset \mathfrak{p}_{0} \cap \mathfrak{h}_{0}=\mathfrak{h}_{\mathfrak{p}_{0}}$ and $\operatorname{Ad}(u) \mathfrak{h}_{\mathfrak{t}_{0}} \subset \mathfrak{h}_{\mathfrak{t}_{0}}$. This shows that $s$ leaves $\mathfrak{h}_{\mathfrak{f}_{0}}$ and $\mathfrak{h}_{\mathfrak{p}_{0}}$ separately invariant. Hence if $\nu$ is any linear function on $\mathfrak{h}_{\mathfrak{p}_{0}}$ (or $\mathfrak{h}_{\mathfrak{t}_{0}}$ or $\left.\mathfrak{h}_{0}\right)$ we can define another such function $s \nu$ by the rule $s \nu(H)=\nu\left(s^{-1} H\right)$. Similarly if $\delta \in \omega_{M_{0} z}$ we can define another class $s^{-1} \delta \in \omega_{M_{0} z}$ by the condition $\xi_{s}{ }^{-1} \delta(h)=\xi_{\delta}\left(h^{s}\right)\left(h \in A_{-}^{0} Z\right)$. That such a class actually exists and is unique is seen as follows. Since $\mathfrak{m}_{0}$ is the centralizer of $\mathfrak{h}_{\mathfrak{p}_{0}}$ in $\mathfrak{f}_{0}$, Ad $(u) \mathfrak{m}_{0}=\mathfrak{m}_{0}$ and $u M_{0} u^{-1}=M_{0}$. Let $\sigma$ be any representation of $M_{0} Z$ in $\delta$. Define a new representation $\sigma^{\prime}$ by the rule 


$$
\sigma^{\prime}(m)=\sigma\left(u m u^{-1}\right) \quad\left(m \in M_{0} Z\right) .
$$

Then $\sigma^{\prime}$ is irreducible and if $\delta^{\prime}$ is its class $\xi_{\delta^{\prime}}(h)=\xi_{\delta}\left(u h u^{-1}\right)=\xi_{\delta}\left(h^{s}\right)\left(h \in A_{-}^{0} Z\right)$. Since every element in $M_{0}$ is conjugate (with respect to $M_{0}$ ) to some element in $A_{-}^{0}$, every class in $\omega_{M_{0} z}$ is completely determined by the restriction of its character on $A_{-}^{0} Z$. Hence $s^{-1} \delta$ is uniquely defined.

Notice that if $\alpha$ is a root, $s \alpha$ is also a root and $s \alpha$ is zero on $\mathfrak{h}_{\mathfrak{p}_{0}}$ if and only if the same holds for $\alpha$. From this it follows immediately that

$$
\Delta_{+}\left(h^{s}\right)=\Delta_{+}(h), \quad \Delta_{-}\left(h^{s}\right)=\Delta_{-}(h) \quad\left(h \in A^{0} Z\right) .
$$

Therefore

$$
\Theta_{\Lambda, \delta}(h)=\left[\Delta_{+}(h)\right]^{-1} \sum_{s \in W} \exp \left\{s(\Lambda+\rho)\left(\log h_{+}\right)\right\} \xi_{s \delta}\left(h_{-}\right)
$$

and we have the following theorem.

THEOREM 2. There exists a positive real constant $c$ with the following property. If $\Lambda$ is any linear function on $\mathfrak{h}_{\mathfrak{p}_{0}}$ and $\delta$ a class in $\omega_{M_{0} z}$, then

$$
T_{\Lambda, \delta}(f)=c \int_{G_{1}} f(x) \Theta_{\Lambda, \delta}(x) d x
$$

for any $f \in C_{c}(G)$. Here $\Theta_{\Lambda, \delta}$ is a continuous function on $G_{1}$ defined uniquely by the following two properties:

$$
\begin{array}{ll}
\Theta_{\Lambda, \delta}\left(y x y^{-1}\right)=\Theta_{\Lambda, \delta}(x) & \left(x \in G_{1}, y \in G\right), \\
\Theta_{\Lambda, \delta}(h)=\left[\Delta_{+}(h)\right]^{-1} \sum_{s \in W} \exp \left\{s(\Lambda+\rho)\left(\log h_{+}\right)\right\} \xi_{s \delta}\left(h_{-}\right) . &
\end{array}
$$

Let $\Lambda^{*}=s(\Lambda+\rho)-\rho$. Then the above theorem shows that $T_{\Lambda^{s}, s \delta}=T_{\Lambda, \delta}$ $\left(\delta \in \omega_{M_{0} z}\right)$. Conversely suppose $\Lambda_{1}, \Lambda_{2}$ are two linear functions on $\mathfrak{h}_{0}$ and $\delta_{1}, \delta_{2}$ two classes in $\omega_{M_{0} Z}$ such that $T_{\Lambda_{1}, \delta_{1}}=T_{\Lambda_{2}, \delta_{2}}$. Then it follows from the above theorem that $\Theta_{\Lambda_{1}, \delta_{1}}=\Theta_{\Lambda_{2}, \delta_{2}}$ on $G_{1}$. Therefore

$$
\sum_{s \in W} \exp \left\{s\left(\Lambda_{1}+\rho\right)\left(\log h_{+}\right)\right\} \xi_{s \delta_{1}}\left(h_{-}\right)=\sum_{s \in W} \exp \left\{s\left(\Lambda_{2}+\rho\right)\left(\log h_{+}\right)\right\} \xi_{s \delta_{2}}\left(h_{-}\right)
$$

for all regular $h \in A^{0} Z$. But since both sides are continuous functions on $A^{0} Z$ and the set of regular elements is dense in $A^{0} Z$, they are equal everywhere. Now the exponentials of distinct linear functions are well known to be linearly independent (see for example [8, Lemma 41]). Hence it follows on putting $h_{-}=1$ that $\Lambda_{2}=\Lambda_{1}^{s_{0}}$ for $s_{0} \in W$. Let $W_{0}$ be the subgroup consisting of all $t \in W$ such that $\Lambda_{2}^{t}=\Lambda_{2}$. Therefore if we compare coefficients of $\exp \left\{\left(\Lambda_{2}+\rho\right)\left(\log h_{+}\right)\right\}$on the two sides we get

$$
\sum_{t \in W_{0}} \xi_{t s_{0} \delta_{1}}\left(h_{-}\right)=\sum_{t \in W_{0}} \xi_{t \delta_{2}}\left(h_{-}\right) \quad\left(h_{-} \in A^{0} Z\right) .
$$

But every element in $M_{0}$ is conjugate to some element in $A_{-}^{0}$ and therefore 


$$
\sum_{t \in W_{0}} \xi_{t s_{0} \delta_{1}}(m)=\sum_{t \in W_{0}} \xi_{t \delta_{2}}(m)
$$

On the other hand it is well known that the characters corresponding to distinct irreducible classes are linearly independent. Hence $\delta_{2}=t s_{0} \delta_{1}$ for some $t \in W_{0}$. Put $s=t s_{0}$. Then $\Lambda_{2}=\Lambda_{1}^{s}$ and $\delta_{2}=s \delta_{1}$. Thus we have the following lemma.

Lemma 12. Let $\Lambda_{1}, \Lambda_{2}$ be two linear functions on $\mathfrak{h}_{\mathfrak{p}_{0}}$ and $\delta_{1}, \delta_{2}$ two classes in $\omega_{M_{0} z}$. Then $T_{\Lambda_{1}, \delta_{1}}=T_{\Lambda_{2}, \delta_{2}}$ if and only if there exists an element $s \in W$ such that $\Lambda_{2}=\Lambda_{1}^{s}$ and $\delta_{2}=s \delta_{1}$.

5. Plancherel formula for complex semisimple Lie groups. We shall now assume that $G$ is a complex semisimple group. We keep to the notation of $\$ 2$ of [6]. Since $G$ is complex, there exists a linear mapping $\Gamma$ of $\mathfrak{t}_{0}$ on $\mathfrak{p}_{0}$ such that $[X, \Gamma(Y)]=\Gamma([X, Y])$ and $[\Gamma(X), \Gamma(Y)]=-[X, Y]\left(X, Y \in \mathfrak{f}_{0}\right)$. We extend $\Gamma$ to a linear mapping of $g_{0}$ onto itself by defining $\Gamma(\Gamma(X))=-X$ $\left(X \in \mathfrak{f}_{0}\right)$. Let $(-1)^{1 / 2}$ be a fixed square root of -1 in $C$. Then if $c=a+(-1)^{1 / 2} b$ $(a, b \in R)$ we put $c * X=a X+b \Gamma(X) \quad\left(X \in \mathfrak{g}_{0}\right)$. Under this multiplication $\mathfrak{g}_{0}$ becomes a Lie algebra over $C$. We shall denote this complex algebra by $g^{*}$. Similarly the algebra $\mathfrak{h}_{0}=\mathfrak{h}_{\mathfrak{t}_{0}}+\mathfrak{h}_{\mathfrak{p}_{\mathfrak{p}}}$, regarded as a (complex) subalgebra of $\mathfrak{g}^{*}$, will be denoted by $\mathfrak{h}^{*}$. Then $\mathfrak{h}^{*}$ is a Cartan subalgebra of $\mathfrak{g}^{*}$. Let $X \rightarrow \operatorname{ad} X$ $\left(X \in \mathfrak{g}^{*}\right)$ denote the adjoint representation of $\mathrm{g}^{*}$ and let $B(X, Y)$ $=\operatorname{sp}(\operatorname{ad} X \operatorname{ad} Y)\left(X, Y \in \mathfrak{g}^{*}\right)$. Given any linear function $\lambda$ on $\mathfrak{h}^{*}$, we denote by $H_{\lambda}$ the unique element in $\mathfrak{h}^{*}$ such that $\lambda(H)=B\left(H, H_{\lambda}\right)$ for all $H \in \mathfrak{h}^{*}$. Let $H_{1}, \cdots, H_{l}$ be a base for $\mathfrak{h}_{\mathfrak{p}_{0}}$ over $R$. Then it is also a base for $\mathfrak{h}^{*}$ over $C$. We shall say that $\lambda$ is real if $H_{\lambda}=\sum_{1 \leqq i \leqq l} c_{i} H_{i}\left(c_{i} \in R\right)$, and furthermore that $\lambda>0$ if $\lambda \neq 0$ and $c_{j}>0$ where $j$ is the least index $(1 \leqq j \leqq l)$ such that $c_{j} \neq 0$. For every root $\alpha$ of $\mathfrak{g}^{*}$ (with respect to $\mathfrak{h}^{*}$ ) we choose an element $X_{\alpha} \neq 0$ in $\mathfrak{g}^{*}$ such that $\left[H, X_{\alpha}\right]=\alpha(H) * X_{\alpha}\left(H \in \mathfrak{h}^{*}\right)$. We can do this in such a way that $B\left(X_{\alpha}, X_{-\alpha}\right)=1$ and $X_{\alpha}-X_{-\alpha},(-1)^{1 / 2} *\left(X_{\alpha}+X_{-\alpha}\right)$ are both in $\mathfrak{f}_{0}$ (The corresponding statement on p. 814 of my earlier note (Proc. Nat. Acad. Sci. U. S. A. vol. 37(1951) pp. 813-818) has wrong signs.) Since every root $\alpha$ is real, $H_{\alpha}=\sum_{i=1}^{l} \alpha^{i} H_{i}\left(\alpha^{i} \in R\right)$. Let $\mathfrak{n}^{*}=\sum_{\alpha \in Q} C * X_{\alpha}$ where $Q$ is the set of all roots $\alpha>0$. Then $\mathfrak{n}^{*}$ is a nilpotent sugalgebra of $g^{*}$ to which there corresponds an analytic subgroup $N$ of $G$. We shall denote by $\mathfrak{n}_{0}$ the space $\mathfrak{n}^{*}$ regarded as a real vector-subspace of $\mathfrak{g}_{0}$.

Let $\mathfrak{g}$ be the complexification of the real algebra $\mathfrak{g}_{0}$. Let $\mathfrak{f}, \mathfrak{p}, \mathfrak{h}, \mathfrak{h}_{\mathfrak{t}}$, and $\mathfrak{h}_{\mathfrak{p}}$ respectively denote the subspaces of $\mathfrak{g}$ spanned by $\mathfrak{f}_{0}, \mathfrak{p}_{0}, \mathfrak{h}_{0}, \mathfrak{h}_{\mathfrak{t}_{0}}$, and $\mathfrak{h}_{\mathfrak{p}_{0}}$ over $C$. We denote by $\gamma$ the isomorphism between $\mathfrak{g}^{*}$ and $\mathfrak{l}$ given by $\gamma(c * X)=c X$ $\left(c \in C, X \in \mathfrak{f}_{0}\right)$. Put

$$
\begin{aligned}
& \gamma_{+}(X)=\left(X-(-1)^{1 / 2} \Gamma(X)\right) / 2, \\
& \gamma_{-}(X)=\left(X+(-1)^{1 / 2} \Gamma(X)\right) / 2
\end{aligned}
$$

and let $\mathfrak{f}_{+}=\gamma_{+}(\mathfrak{f}), \mathfrak{f}_{-}=\gamma_{-}(\mathfrak{f})$. (Here we have extended $\Gamma$ on $\mathfrak{g}$ by linearity.) 
Then $\mathfrak{f}_{+}$and $\mathfrak{t}_{-}$are ideals in $\mathfrak{g}$ and $\mathfrak{g}$ is their direct sum. Moreover $\gamma_{+}$and $\gamma_{-}$ are isomorphisms of $\mathfrak{t}$ on $\mathfrak{l}_{+}$and $\mathfrak{t}_{-}$respectively. Now $\mathfrak{h}=\gamma_{+}\left(\mathfrak{h}_{\mathfrak{t}}\right)+\gamma_{-}\left(\mathfrak{h}_{\mathfrak{t}}\right)$. Let $\lambda, \mu$ be two linear functions on $\mathfrak{h}^{*}$. Then we denote by $(\lambda, \mu)$ the linear function $\nu$ on $\mathfrak{h}$ defined as follows:

$$
\begin{aligned}
& \nu\left(\gamma_{+}(\gamma(H))\right)=\lambda(H), \\
& \nu\left(\gamma_{-}(\gamma(H))\right)=\mu(H)
\end{aligned}
$$$$
\left(H \in \mathfrak{h}^{*}\right) .
$$

It is easy to verify that

$$
\begin{aligned}
& \nu(H)=\lambda(H)+\mu(H) \\
& \nu(H)=\lambda(H)-\mu(H)
\end{aligned}
$$

if $H \in \mathfrak{h}_{\mathfrak{t}_{0}}$, if $H \in \mathfrak{h}_{\mathfrak{p}_{0}}$.

Put $\lambda_{+}=(\lambda, 0)$ and $\lambda_{-}=(0,-\lambda)$. Then if $\lambda$ is real (in the sense described above) $\lambda_{+}(H)=\lambda(H)$ and $\lambda_{-}(H)=\operatorname{conj} \lambda(H)$ for $H \in \mathfrak{h}_{0}$. Therefore, in particular, for every root $\alpha$ (of $\mathfrak{g}^{*}$ with respect to $\mathfrak{h}^{*}$ ) we get two linear functions $\alpha_{+}, \alpha_{-}$on $\mathfrak{h}$ and if we put $X_{\alpha}^{+}=\gamma_{+}\left(\gamma\left(X_{\alpha}\right)\right), X_{\alpha}^{-}=\gamma_{-}\left(\gamma\left(X_{\alpha}\right)\right)$, we have

$$
\left[H, X_{\alpha}^{+}\right]=\alpha_{+}(H) X_{\alpha}^{+}, \quad\left[H, \overline{X_{\alpha}^{-}}\right]=\alpha_{-}(H) \overline{X_{\alpha}} \quad(H \in \mathfrak{h}) .
$$

Notice that $\alpha_{+} \neq \alpha_{-}$since $\alpha_{+}$vanishes on $\gamma_{-}\left(\mathfrak{h}_{t}\right)$ while $\alpha_{-}$vanishes on $\gamma_{+}\left(\mathfrak{h}_{t}\right)$ and neither of them is zero. Hence for each root $\alpha$ of $g^{*}$ we get two distinct roots $\alpha_{+}$and $\alpha_{-}$of $\mathfrak{g}$. Moreover if we take $\left(H_{1}, \cdots, H_{l},(-1)^{1 / 2} \Gamma\left(H_{1}\right), \cdots\right.$, $\left.(-1)^{1 / 2} \Gamma\left(H_{l}\right)\right)$ as an ordered base for $\mathfrak{h}_{\mathfrak{p}_{0}}+(-1)^{1 / 2} \mathfrak{h}_{\mathfrak{t}_{0}}$ over $R$ and define the sets $P, P_{+}$, and $P_{-}$of positive roots of $\mathfrak{g}$ with respect to this base (see $[6, \S 2]$ ), we find that if $\alpha \in Q, \alpha_{+}$and $\alpha_{-}$are both in $P$. In view of the isomorphisms $\gamma_{+}$and $\gamma_{-}$it is clear that every root of $g$ is of the form $\pm \alpha_{ \pm}(\alpha \in Q)$. Hence every root in $P$ is the form $\alpha_{ \pm}$for some $\alpha \in Q$. Moreover since $\alpha$ is complex-linear, it cannot vanish on $\mathfrak{h}_{\mathfrak{p}_{0}}$. Therefore the set $P_{-}$is empty and $P=P_{+}$. Now let $X_{\alpha}=X_{\alpha}^{\prime}+\Gamma\left(X_{\alpha}^{\prime \prime}\right)\left(X_{\alpha}^{\prime}, X_{\alpha}^{\prime \prime} \in \mathfrak{f}_{0}\right)$. Then $\gamma\left(X_{\alpha}\right)=X_{\alpha}^{\prime}+(-1)^{1 / 2} X_{\alpha}^{\prime \prime}$ and it is easily verified that

$$
\begin{aligned}
& X_{\alpha}^{+}=\left(X_{\alpha}-(-1)^{1 / 2} \Gamma\left(X_{\alpha}\right)\right) / 2, \\
& X_{\alpha}^{-}=\left(X_{\alpha}+(-1)^{1 / 2} \Gamma\left(X_{\alpha}\right)\right) / 2 .
\end{aligned}
$$

Hence $X_{\alpha}=X_{\alpha}^{+}+X_{\alpha}^{-}$and therefore $\mathfrak{n}^{*}=\mathfrak{n}_{0} \subset \mathfrak{g}_{0} \cap\left\{\sum_{\alpha \in Q}\left(C X_{\alpha}^{+}+C X_{\alpha}^{-}\right)\right\}$. Since $\operatorname{dim}_{R} \mathfrak{n}_{0}=2 \operatorname{dim}_{C} \mathfrak{n}^{*}, \operatorname{dim}_{R} \mathfrak{n}_{0}$ is equal to the number of roots in $P_{+}=P$. Hence $\mathfrak{n}_{0}=\mathfrak{g}_{0} \cap\left\{\sum_{\alpha \in Q}\left(C X_{\alpha}^{+}+C X_{\alpha}^{-}\right)\right.$.

If $f$ is a complex-valued differentiable function of two real variables $x, y$ and $z=x+(-1)^{1 / 2} y$ we write

$$
\frac{\partial}{\partial z} f=\frac{1}{2}\left(\frac{\partial}{\partial x}-(-1)^{1 / 2} \frac{\partial}{\partial y}\right) f, \quad \frac{\partial}{\partial \bar{z}} f=\frac{1}{2}\left(\frac{\partial}{\partial x}+(-1)^{1 / 2} \frac{\partial}{\partial y}\right) f
$$

where the bar denotes complex conjugate. Put $\rho=(1 / 2) \sum_{\alpha \in Q} \alpha$ and let $K$ and $A_{+}$be the analytic subgroups of $G$ corresponding to $\mathfrak{l}_{0}$ and $\mathfrak{h}_{p_{0}}$ respec- 
tively. Then $K$ is compact. Moreover $\mathfrak{c}_{0}=\{0\}$ in the present case and therefore $D=\{1\}$. Let $d u$ and $d n$ denote the Haar measures on $K$ and $N$ respectively. We assume $\int_{K} d u=1$. The following theorem is the principal step in the proof of the Plancherel formula for $G$ (see Gelfand and Naimark [3, p. 198]).

Theorem 3. Put $H_{a}=\sum_{1 \leqq i \leqq l} a_{i} * H_{i}\left(a_{i} \in C\right)$ and

$$
D_{\alpha}=\sum_{1 \leqq i \leqq l} \alpha^{i} \frac{\partial}{\partial a_{i}}, \quad \bar{D}_{\alpha}=\sum_{1 \leqq i \leqq l} \alpha^{i} \frac{\partial}{\partial \bar{a}_{i}} \quad(\alpha \in Q) .
$$

Then if $d n$ is suitably normalised we have

$$
f(1)=\lim _{H_{a} \rightarrow 0} \prod_{\alpha \in Q} D_{\alpha} \bar{D}_{\alpha}\left\{\exp \left\{\rho\left(H_{a}\right)+\overline{\rho\left(\overline{H_{a}}\right)}\right\} \int_{K \times N} f\left(u\left(\exp H_{a}\right) n u^{-1}\right) d u d n\right\}
$$

for every $f \in C_{c}^{\infty}(G)$.

The proof depends on the theory of Fourier transforms for functions on $\mathfrak{g}_{\mathbf{0}}$. Let $C_{c}^{\infty}\left(g_{0}\right)$ be the class of all complex-valued functions on $g_{0}$ which are everywhere indefinitely differentiable and which vanish outside a compact set. Put

$$
X=\sum_{1 \leqq i \leqq l} a_{i} * H_{i}+\sum_{\alpha \in Q}\left(z_{\alpha} * X_{\alpha}+\overline{z_{\alpha} * X_{-\alpha}}\right)
$$

where $a_{i}, z_{\alpha}, z_{\alpha}^{-}(1 \leqq i \leqq l, \alpha \in Q)$ are independent complex variables. For any complex variable $z=x+(-1)^{1 / 2} y(x, y \in R)$ let $d \mu(z)$ denote the Euclidean measure $d x d y$ on the corresponding complex plane. Let $F$ be a function in $C_{c}^{\infty}\left(\mathfrak{g}_{0}\right)$. Put

$$
g(Y)=(2 \pi)^{-n} \int_{\mathfrak{B}_{0}} \exp \left\{(-1)^{1 / 2} \Re(B(X, Y))\right\} F(X) d X
$$

where $n=(1 / 2) \operatorname{dim}_{R} \mathrm{~g}_{0}, d X=\prod_{1 \leqq i \leqq l} d \mu\left(a_{i}\right) \prod_{\alpha \in Q} d \mu\left(z_{\alpha}\right) d \mu\left(z_{\alpha}^{-}\right)$and $\Re c(c \in C)$ denotes the real part of $c$. Then if we assume, as we may, that $B\left(H_{i}, H_{j}\right)$ $=\delta_{i j}, 1 \leqq i, j \leqq l$ ( $\delta_{i j}$ is the usual Kronecker symbol), it follows from the theory of Fourier transforms that $\int_{\mathfrak{B}_{0}}|g(X)| d X<\infty$ and

$$
F(0)=(2 \pi)^{-n} \int_{\mathbb{g}_{0}} g(X) d X .
$$

Now suppose $F(\operatorname{Ad}(u) X)=F(X)$ for all $u \in K$ and $X \in g_{0}$. We know that the bilinear form $B(X, Y)$ is invariant under the adjoint representation of $G$ and $d(\operatorname{Ad}(x) X)=d X(x \in G)$ since det $\operatorname{Ad}(x)=1$. We can now transform the integral $\int_{\mathfrak{g}_{0}} g(X) d X$ in another form. Let $d H$ and $d Z$ denote the Euclidean measures on $\mathfrak{h}_{0}$ and $\mathfrak{n}_{0}$ respectively. Then we have the following lemma.

Lemma 13. It is possible to normalise the measures $d Z$ and $d H$ in such a way 
that

$$
\int_{\mathfrak{B}_{0}} g(X) d X=\int_{\mathfrak{G}_{0}+\mathfrak{n}_{0}} \prod_{\alpha \in Q}|\alpha(H)|^{2} g(Z+H) d Z d H
$$

for any measurable function $g(X)$ on $g_{0}$ such that $g(\operatorname{Ad}(u) X)=g(X)(u \in K$, $\left.X \in g_{0}\right)$ and $\int_{g_{0}}|g(X)| d X<\infty$.

Let $g_{1}$ be the set of all regular elements in $g_{0}$. Then we know (see Chevalley [2]) that every $X \in \mathfrak{g}_{1}$ is conjugate under $G$ to some $H \in \mathfrak{h}_{0}$. Since the set of singular elements in $g_{0}$ is of measure zero,

$$
\int_{\mathfrak{B}_{0}} g(X) d X=\int_{\mathfrak{g}_{1}} g(X) d X
$$

and Lemma 9 is applicable. Now $P_{-}$is empty and it follows from our earlier remarks that

$$
\prod_{\beta \in P_{+}}|\beta(H)|=\prod_{\alpha \in Q}|\alpha(H)|^{2} \quad\left(H \in \mathfrak{h}_{0}\right) .
$$

Moreover the set of singular elements in $\mathfrak{h}_{0}$ is also of measure zero (with respect to the Euclidean measure $d H$ on $\mathfrak{h}_{0}$ ). The above lemma is therefore an immediate consequence of Lemma 9.

$F$ and $g$ being as above, consider the function $F^{\prime}=\prod_{\alpha \in Q} D_{\alpha} \bar{D}_{\alpha} F$. Its Fourier transform $g^{\prime}$ is given by

$$
g^{\prime}(Y)=(2 \pi)^{-n} \int_{\mathfrak{g}_{0}} \exp \left\{(-1)^{1 / 2} \mathfrak{R}(B(X, Y))\right\} F^{\prime}(X) d X \quad\left(Y \in \mathfrak{g}_{0}\right) .
$$

Let $X=\sum_{i=1}^{l} a_{i} * H_{i}+\sum_{\alpha \in Q}\left(z_{\alpha} * X_{\alpha}+z_{\alpha}^{-} * X_{-\alpha}\right)$ and $Y=\sum_{i=1}^{l} b_{i} * H_{i}$ $+\sum_{\alpha \in Q}\left(w_{\alpha} * X_{\alpha}+w_{\alpha}^{-} * X_{-\alpha}\right)$ where $\left(a, z, z^{-}, b, w, w^{-}\right)$are all independent complex variables. Put $F(X)=F\left(a, z, z^{-}\right), g(Y)=g\left(b, w, w^{-}\right)$, and $g^{\prime}(Y)$ $=g^{\prime}\left(b, w, w^{-}\right)$. Then

$$
\begin{aligned}
g^{\prime}\left(b, w, w^{-}\right)= & (2 \pi)^{-n} \int \exp \left\{(-1)^{1 / 2} \Re\left(\sum_{i=1}^{l} a_{i} b_{i}+\sum_{\alpha \in Q}\left(z_{\alpha} w_{\alpha}^{-}+z_{\alpha}^{-} w_{\alpha}\right)\right)\right\} \\
& \cdot \prod_{\alpha \in Q} D_{\alpha} \bar{D}_{\alpha} F\left(a, z, z^{-}\right) d \mu(a) d \mu(z) d \mu\left(z^{-}\right)
\end{aligned}
$$

where

$$
d \mu(a)=\prod_{i=1}^{l} d \mu\left(a_{i}\right), \quad d \mu(z)=\prod_{\alpha \in Q} d \mu\left(z_{\alpha}\right), \quad d \mu\left(z^{-}\right)=\prod_{\alpha \in Q} d \mu\left(z_{\alpha}^{-}\right) .
$$

Hence by partial integration

$$
g^{\prime}\left(b, w, w^{-}\right)=\prod_{\alpha \in Q}\left|\alpha\left(H_{b}\right)\right|^{2} b\left(g, w, w^{-}\right)
$$


where $H_{b}=\sum_{i=1}^{l} b_{i} * H_{i}$. Therefore

$$
\begin{aligned}
& \int \prod_{\alpha \in Q}\left|\alpha\left(H_{a}\right)\right|^{2} g(a, z, 0) d \mu(a) d \mu(z) \\
&=\int g^{\prime}(a, z, 0) d \mu(a) d \mu(z) \\
&=(2 \pi)^{-n} \int d \mu(a) d \mu(z) \int \exp \left\{(-1)^{1 / 2} \Re\left(\sum_{i=1}^{l} a_{i} b_{i}+\sum_{\alpha \in Q} z_{\alpha} w_{\alpha}^{-}\right)\right\} \\
& \cdot F^{\prime}\left(b, w, w^{-}\right) d \mu(b) d \mu(w) d \mu\left(w^{-}\right) \\
&=(2 \pi)^{-n+r} \int F^{\prime}(0, w, 0) d \mu(w)
\end{aligned}
$$

from the theory of Fourier transforms. Here $r$ is the complex dimension of $\mathfrak{h}^{*}+\mathfrak{n}^{*}=\mathfrak{h}_{0}+\mathfrak{n}_{0}$. This shows that

$$
\begin{aligned}
F(0) & =(2 \pi)^{-n} \int_{\mathbf{B}_{0}} g(X) d X \\
& =c(2 \pi)^{-n} \int \prod_{\alpha \in Q}\left|\alpha\left(H_{a}\right)\right|^{2} g(a, z, 0) d \mu(a) d \mu(z) \quad \text { (from Lem } \\
& =c(2 \pi)^{-n+r} \int F^{\prime}(0, w, 0) d \mu(w) \\
& =c(2 \pi)^{-n+r} \int \lim _{H_{a} \rightarrow 0}\left\{\prod_{\alpha \in Q} D_{\alpha} \bar{D}_{\alpha} F\left(H_{\alpha}+\sum_{\alpha \in Q} z_{\alpha} * X_{\alpha}\right)\right\} d \mu(z)
\end{aligned}
$$

where $c$ is a positive real constant independent of $F$. Since $F$ vanishes outside a compact set it follows easily that

$$
\begin{aligned}
\int \lim _{H_{a} \rightarrow 0}\left\{\prod_{\alpha \in Q} D_{\alpha} \bar{D}_{\alpha} F\left(H_{a}+\sum_{\alpha \in Q} z_{\alpha} X_{\alpha}\right)\right\} d \mu(z) \\
=\lim _{H_{a} \rightarrow 0} \prod_{\alpha \in Q} D_{\alpha} \bar{D}_{\alpha}\left\{\int F\left(H_{a}+\sum_{\alpha \in Q} z_{\alpha} * X_{\alpha}\right) d \mu(z)\right\} .
\end{aligned}
$$

Thus we have the following result.

LEMMa 14. There exists a positive real constant $c$ with the following property: For any $F \in C_{c}^{\infty}\left(\mathfrak{g}_{0}\right)$ such that $F(\operatorname{Ad}(u) X)=F(X)\left(u \in K, X \in \mathfrak{g}_{0}\right)$,

$$
F(0)=\lim _{H_{a} \rightarrow 0} c \prod_{\alpha \in Q} D_{\alpha} \bar{D}_{\alpha} \int F\left(H_{a}+\sum_{\alpha \in Q} z_{\alpha} * X_{\alpha}\right) d \mu(z) .
$$

Now we come to the proof of Theorem 3. Let $f$ be a function in $C_{c}^{\infty}(G)$. 
Put

$$
f_{1}(x)=\int_{K} f\left(u x u^{-1}\right) d u \quad(x \in G) .
$$

Then $f_{1}$ is also in $C_{c}^{\infty}(G)$. Let $F_{1}(X)=f_{1}(\exp X)\left(X \in g_{0}\right)$. Choose a compact neighbourhood $U$ of zero in $\mathfrak{h}_{0}$ corresponding to Lemma 11 . Let $\gamma$ be the carrier of $F_{1}$ (i.e. the smallest closed set outside which $F_{1}$ is zero). Then $\gamma_{1}=\gamma \cap\left(U+\mathfrak{n}_{0}\right)$ is the complete inverse image in $U+\mathfrak{n}_{0}$ (under the exponential mapping) of the intersection of the carrier of $f_{1}$ with the closed set $\exp \left(U+\mathfrak{n}_{0}\right)$ (see corollary to Lemma 11). Hence $\gamma_{1}$ is compact. Then $E=\bigcup_{u \in K} \operatorname{Ad}(u) \gamma_{1}$ is also compact. Moreover the exponential mapping is regular on $\gamma_{1}$ and therefore on $E$. Since $E$ is compact, it is clear that there exists a compact neighbourhood $V_{1}$ of $E$ in $g_{0}$ such that the exponential mapping is everywhere regular on $V_{1}$. Put $V=U_{u \in K}$ Ad $(u) V_{1}$. Then $V$ is still compact and the exponential mapping is regular on $V$. Let $V^{\prime}$ be an open neighbourhood of $V$ such that the exponential mapping is still regular on $V^{\prime}$. We may assume that the closure of $V^{\prime}$ is compact. Select a function $\phi \in C_{c}^{\infty}\left(\mathrm{g}_{0}\right)$ such that $\phi=1$ on $V$ and $\phi=0$ outside $V^{\prime}$.

For any $X \in \mathfrak{g}_{0}$ consider the endomorphism $\left(1-e^{-\mathrm{ad} X}\right) / \mathrm{ad} X$ of $\mathfrak{g}_{0}$. (Here $X \rightarrow \operatorname{ad} X$ is the adjoint representation of the real algebra $g_{0}$.) Since the exponential mapping is regular on $V^{\prime}$, $\operatorname{det}\left(\left(1-e^{-\operatorname{ad} X}\right) / \operatorname{ad} X\right) \neq 0$ on $V^{\prime}$. Therefore the function $\left|\operatorname{det}\left(\left(1-e^{-\mathrm{ad} X}\right) / \operatorname{ad} X\right)\right|^{1 / 2}$ is indefinitely differentiable on $V^{\prime}$. Put

$$
F_{2}(X)=F_{1}(X) \phi(X)\left|\operatorname{det}\left(\left(1-e^{-\operatorname{ad} X}\right) / \operatorname{ad} X\right)\right|^{1 /}
$$

Since $\phi$ is zero outside $V^{\prime}$ it follows that $F_{2} \in C_{c}^{\infty}\left(g_{0}\right)$. Now let

$$
F(X)=\int_{K} F_{2}(\operatorname{Ad}(u) X) d u \quad\left(X \in g_{0}\right) .
$$

If $X \in V$,

$$
F_{2}(\operatorname{Ad}(u) X)=F_{1}^{\prime}(\operatorname{Ad}(u) X)=F_{1}^{\prime}(X)
$$

where

$$
F_{1}^{\prime}(X)=F_{1}(X)\left|\operatorname{det}\left(\frac{1-e^{-\mathrm{ad} X}}{\operatorname{ad} X}\right)\right|^{1 / 2} .
$$

Hence $F(X)=F_{1}^{\prime}(X)$ if $X \in V$. Now suppose $X \in U+\mathfrak{n}_{0}$ but $X \notin V$. Then $X \notin \gamma$ and since $\gamma=\operatorname{Ad}(u) \gamma(u \in K), \operatorname{Ad}(u) X \notin \gamma$ for any $u \in K$. Hence

$$
F(X)=\int_{K} F_{2}(\operatorname{Ad}(u) X) d u=0=F_{1}^{\prime}(X) .
$$

This proves that $F=F_{1}^{\prime}$ on $U+\mathfrak{n}_{0}$. 
Since $F_{2} \in C_{c}^{\infty}\left(g_{0}\right)$ the same holds for $F$. Moreover $F(\operatorname{Ad}(u) X)=F(X)$ $\left(u \in K, X \in \mathrm{g}_{0}\right)$. Therefore from Lemma 14

$$
\begin{aligned}
F(0) & =c \lim _{a \rightarrow 0} \prod_{\alpha \in Q} D_{\alpha} \bar{D}_{\alpha} \int F\left(H_{a}+\sum_{\alpha \in Q} z_{\alpha} X_{\alpha}\right) d \mu(z) \\
& =c \lim _{a \rightarrow 0} \prod_{\alpha \in Q} D_{\alpha} \bar{D}_{\alpha} \int_{\mathfrak{n}_{0}} F_{1}^{\prime}\left(H_{a}+Z\right) d Z
\end{aligned}
$$

where $d Z=d \mu(z) \quad\left(Z=\sum_{\alpha \in Q} z_{\alpha} * X_{\alpha}\right)$ is the Euclidean measure on $\mathfrak{n}_{0}$. But

$$
\begin{aligned}
F_{1}^{\prime}(H+Z) & =F_{1}(H+Z)\left|\operatorname{det}\left(\frac{1-e^{-\mathrm{ad}(H+Z)}}{\operatorname{ad}(H+Z)}\right)\right|^{1 / 2} \\
& =F_{1}(H+Z)\left|\operatorname{det}\left(\frac{1-e^{-\mathrm{ad} H}}{\operatorname{ad} H}\right)\right|^{1 / 2} \quad\left(H \in \mathfrak{h}_{0}, Z \in \mathfrak{n}_{0}\right)
\end{aligned}
$$

and

$$
\begin{aligned}
\operatorname{det}\left(\frac{1-e^{-\mathrm{ad} H}}{\operatorname{ad} H}\right) & =\prod_{\beta \in P_{+}} \frac{1-e^{-\beta(H)}}{\beta(H)} \prod_{\beta \in P_{+}} \frac{1-e^{\beta(H)}}{\beta(H)} \\
& =\left\{\prod_{\beta \in P_{+}} \frac{e^{\beta(H) / 2}-e^{-\beta(H) / 2}}{\beta(H)}\right\}^{2} .
\end{aligned}
$$

Therefore

$$
F_{1}^{\prime}(H+Z)=F_{1}(H+Z) \Delta_{+}(H)
$$

where

$$
\Delta_{+}(H)=\left|\prod_{\beta \in P_{+}} \frac{e^{\beta(H) / 2}-e^{-\beta(H) / 2}}{\beta(H)}\right|
$$

Now let $H \in U$. Then the mapping $\phi: Z \rightarrow h^{-1} \exp (H+Z)(h=\exp H)$ is a topological and regular mapping of $\mathfrak{n}_{0}$ onto $N$ (see Lemmas 10 and 11) and

$$
\operatorname{det}(d \phi)_{z}=\operatorname{det}\left(\frac{1-e^{-D}}{D}\right)
$$

where $D$ is the restriction of ad $(H+Z)$ on $\mathfrak{n}_{0}$. Hence

$$
\left|\operatorname{det}(d \phi)_{Z}\right|=\exp \{-\rho(H)-\overline{\rho(H)}\} \Delta_{+}(H) .
$$

Therefore

$$
\Delta_{+}(H) \int_{\mathfrak{n}_{0}} F_{1}(H+Z) d Z=\exp \{\rho(H)+\overline{\rho(H)}\} \int_{N} f_{1}((\exp H) n) d n
$$


where $d n=d Z(n=\exp Z)$ is the Haar measure on $N$. This proves that

$$
\int_{\mathfrak{n}_{0}} F_{1}^{\prime}(H+Z) d Z=\exp \{\rho(H)+\overline{\rho(H)}\} \int_{N} f_{1}((\exp H) n) d n \quad(H \in U)
$$

and therefore

$$
\left.F(0)=c \lim _{H_{a} \rightarrow 0} \prod_{\alpha \in Q} D_{\alpha} \bar{D}_{\alpha}\left\{\exp \left\{\rho\left(H_{a}\right)+\overline{\rho\left(H_{a}\right.}\right)\right\} \int_{N} f_{1}\left(\left(\exp H_{a}\right) n\right) d n\right\} .
$$

But

$$
F(0)=F_{1}^{\prime}(0)=f_{1}(1)=f(1)
$$

since $0 \in V$. Hence the theorem.

We shall now obtain the Plancherel formula from Theorem 3. In the present case $\mathfrak{h}_{\mathfrak{t}_{0}}=\mathfrak{m}_{0}$ (in the notation of $\S \S 3$ and 4) and $\mathfrak{h}_{\mathfrak{t}_{0}}$ is a maximal abelian subalgebra of $\mathfrak{t}_{0}$. Hence $A_{-}^{0}$ is a maximal abelian subgroup of $K$ and it is its own centralizer in $K$ (see A. Weil [12]). Now the adjoint representation can be regarded as a complex representation of $G$ on $\mathfrak{g}^{*}$. Let $u$ be an element in the centralizer of $A_{+}$in $K$. Then Ad $(u)$ leaves every point in $\mathfrak{h}_{\mathfrak{p}_{0}}$ fixed. But since $\operatorname{Ad}(u)$ is an endomorphism of $\mathfrak{g}^{*}$ over $C$, it leaves every point in $\mathfrak{h}_{\mathfrak{t}_{0}}$ also fixed. This proves that $M=A_{-}^{0}=A_{-}=M_{0} Z$ in the present case. Let $A_{-}^{\prime}$ be the normalizer of $A_{-}$and $K$. Then the above argument shows that $A_{-}^{\prime}$ is also the normalizer of $A^{0}=A_{+} A_{-}^{0}$ in $K$ and therefore $W=A^{\prime} / A$ $=A_{-}^{\prime} / A_{-}$in the notation of $\S 4$. Moreover for any $s \in W$, the mapping $H \rightarrow s H\left(H \in \mathfrak{h}_{0}\right)$ is an endomorphism of $\mathfrak{h}^{*}$ over $C$.

Since $A_{-}$is a torus we can choose the base $H_{1}, \cdots, H_{l}$ of $\mathfrak{h}_{\mathfrak{p}_{0}}$ over $R$ in such a way that $\exp H_{a}=1$ if and only if $(-1)^{1 / 2} a_{i} / 2 \pi, 1 \leqq i \leqq l$, are all rational integers. For any $h \in A=A_{+} A_{-}$we denote by $h_{+}$and $h_{-}$the unique elements in $A_{+}$and $A_{-}$respectively such that $h=h_{+} h_{-}$. Let $\mathfrak{F}_{+}$be the set of all linear functions $\nu$ on $\mathfrak{h}^{*}$ which take real values on $\mathfrak{h}_{\mathfrak{p}_{0}}$. Moreover let $\mathfrak{F}$ - be the set of all linear functions $\lambda$ on $\mathfrak{h}^{*}$ such that $\lambda\left(H_{i}\right), 1 \leqq i \leqq l$, are all rational integers. For every $\lambda \in F_{-}$we can define a character $\xi_{\lambda}$ of $A_{-}$by the rule $\xi_{\lambda}(\exp H)=e^{\lambda(H)}\left(H \in \mathfrak{h}_{\mathfrak{t}_{0}}\right)$ and conversely every character of $A_{-}$can be obtained from some $\lambda \in \mathfrak{F}_{-}$in this way. Put

$$
\xi_{\nu, \lambda}(h)=\exp \left\{(-1)^{1 / 2} \nu\left(\log h_{+}\right)\right\} \xi_{\lambda}\left(h_{-}\right) \quad\left(\nu \in \mathfrak{F}_{+}, \lambda \in \mathfrak{F}_{-}, h \in A\right)
$$

and

$$
S_{\nu, \lambda}(f)=\int_{K \times A \times N} f\left(u h n u^{-1}\right) \xi_{\nu, \lambda}(h) \exp \left\{2 \rho\left(\log h_{+}\right)\right\} d u d h d n \quad\left(f \in C_{c}^{\infty}(G)\right)
$$

where $d h$ is the Haar measure on $A$. It is easy to verify that if $h=\exp H$ $\left(H \in \mathfrak{h}_{0}\right)$ then $\rho(H)+\overline{\rho(H)}=2 \rho\left(\log h_{+}\right)$and

$$
\lim _{H_{\alpha} \rightarrow 0} \prod_{\alpha \in Q} D_{\alpha} \bar{D}_{\alpha} \xi_{\nu, \lambda}\left(\exp H_{a}\right)=\prod_{\alpha \in Q}\left|(-1)^{1 / 2} \nu\left(H_{\alpha}\right)+\lambda\left(H_{\alpha}\right)\right|^{2}=m(\nu, \lambda) \quad \text { (say). }
$$


Now $\mathfrak{F}_{+}$and $\mathfrak{F}_{-}$are groups under ordinary addition of functions and it is clear that they may be considered as character groups of $A_{+}$and $A_{-}$respectively. Let $d \nu$ denote the Haar measure on $\mathfrak{F}_{+}$. Then it is the Euclidean measure on the real vector space $\mathfrak{F}_{+}$. A simple application of the theory of Fourier transforms to the abelian Lie group $A$ now shows that

$$
\begin{aligned}
&\left.\lim _{H_{a} \rightarrow 0} \prod_{\alpha \in Q} D_{\alpha} \bar{D}_{\alpha}\left\{\exp \left\{\rho\left(H_{a}\right)+\overline{\rho\left(H_{a}\right.}\right)\right\} \int f\left(u \exp H_{a} n u^{-1}\right) d u d n\right\} \\
&=\sum_{\lambda \in \mathfrak{F}_{-}} \int_{\mathfrak{F}_{+}} m(\nu, \lambda) S_{\nu, \lambda}(f) d \nu \quad\left(f \in C_{c}^{\infty}(G)\right)
\end{aligned}
$$

provided $d \nu$ is suitably normalised. Here the series on the right is absolutely convergent since the function

$$
g(h)=\exp \left\{2 \rho\left(\log h_{+}\right)\right\} \int f\left(u h \cdot n u^{-1}\right) d u d n
$$

is everywhere indefinitely differentiable on $A$ and vanishes outside a compact set and $S_{\nu, \lambda}(f)$ is the Fourier transform of $g$. Now $\rho^{\prime}(H)=2^{-1} \sum_{\beta \in P} \beta(H)$ $=2 \rho(H) \quad\left(H \in \mathfrak{h}_{\mathfrak{p}_{0}}\right)$. Hence it follows from Theorem 1 that $S_{\nu, \lambda}(f)=T_{\Lambda, \delta}(f)$ where $\Lambda(H)+2 \rho(H)=(-1)^{1 / 2} \nu(H)\left(H \in \mathfrak{h}_{\mathfrak{p}_{0}}\right)$ and $\delta$ is the class of the onedimensional representation $h \rightarrow \xi_{\lambda}(h)\left(h \in A_{-}\right)$of $A_{-}=M=M_{1}$. Moreover since $\Lambda+\rho^{\prime}$ takes pure imaginary values on $\mathfrak{h}_{\mathfrak{p}_{0}}, S_{\nu, \lambda}$ is a unitary character of $G$. Finally, in view of Lemma $12, S_{\nu_{1}, \lambda_{1}}=S_{\nu_{2}, \lambda_{2}}\left(\nu_{1}, \nu_{2} \in \mathfrak{F}_{+} ; \lambda_{1}, \lambda_{2} \in \mathfrak{F}_{-}\right)$if and only if $\nu_{2}=s \nu_{1}, \lambda_{2}=s \lambda_{1}$ for some $s \in W$. Now we need the following theorem.

THEOREM 4. Given any $\lambda \in \mathfrak{F}_{-}$we can find a subset $V_{\lambda}$ of $\mathfrak{F}_{+}$of measure zero such that the character $S_{\nu, \lambda}$ is irreducible for all $\nu$ in $\mathfrak{F}_{+}$outside $V_{\lambda}$.

If we assume this theorem for a moment, we can derive the Plancherel formula as follows. Let $V=U_{\lambda \in F_{-}}$. Since $\mathfrak{F}_{-}$is a countable set, $V$ is still a set of measure zero. Let $V^{\prime}$ be the set of all $\nu \in \mathfrak{F}_{+}$such that $\nu=s \nu$ for some $s \neq 1$ in $W$. Then $V^{\prime}$ is a closed nowhere dense subset of $\mathfrak{F}_{+}$and its measure is zero. Let $\mathfrak{F}_{+}^{\prime}$ be a connected component of the complement of $V^{\prime}$ in $\mathfrak{F}_{+}$. Then $\mathfrak{F}_{+}^{\prime}$ is an open subset of $\mathfrak{F}_{+}$and it is known (see Weyl [13]) that for every $\nu \in \mathfrak{F}_{+}$which is not in $V^{\prime}$ there exists a unique $s \in W$ such that $s \nu \in \mathfrak{F}_{+}$. Since $S_{s \nu, s \lambda}(f)=S_{\nu, \lambda}(f)$ it is clear that

$$
\sum_{\lambda \in \mathfrak{F}_{-}} \int_{\mathfrak{F}_{+}} m(\nu, \lambda) S_{\nu, \lambda}(f) d \nu=\sum_{\lambda \in \mathfrak{F}_{-}} w \int_{\mathfrak{F}_{+}^{0}} m(\nu, \lambda) S_{\nu, \lambda}(f) d \nu \quad\left(f \in C_{c}^{\infty}(G)\right)
$$

where $\mathfrak{F}_{+}^{0}$ is the complement of $V$ in $\mathfrak{F}_{+}^{\prime}$ and $w$ is the order of $W$. Therefore we get

$$
f(1)=\sum_{\lambda \in \mathfrak{F}} w \int_{\mathfrak{F}^{0}+} m(\nu, \lambda) S_{\nu, \lambda}(f) d \nu \quad\left(f \in C_{c}^{\infty}(G)\right)
$$


from Theorem 3. Now the characters $S_{\nu, \lambda}\left(\nu \in \mathfrak{F}_{+}^{0}, \lambda \in \mathfrak{F}_{-}\right)$are all distinct and they are unitary and irreducible. Hence if $\varepsilon$ is the set of all equivalenceclasses of irreducible unitary irreducible representations of $G$, we get a 1-1 mapping of $\mathfrak{F}_{+}^{0} \times \mathfrak{F}_{-}$into $\mathcal{E}$ if we assign to each $(\nu, \lambda)$ the unique class $\omega(\nu, \lambda)$

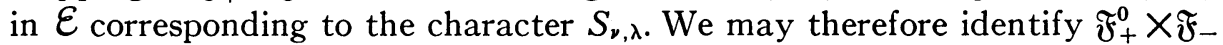
with its image under this mapping and thus regard $\mathfrak{F}_{+}^{0} \times \mathfrak{F}_{-}$as a subset of $\mathcal{E}$. Let $d \mu$ be the discrete measure on $\mathfrak{F}_{-}$- which assigns to every point in $\mathfrak{F}_{-}$the mass $w$. Then we can define a (positive) measure $d \omega$ on $\varepsilon$ as follows. Let $F$ be a subset of $\varepsilon$. We say that $F$ is measurable if $F_{0}=F \cap\left(\mathfrak{F}_{+}^{0} \times \mathfrak{F}_{-}\right)$is measurable in $\mathfrak{F}_{+}^{0} \times \mathfrak{F}_{-}$and in case this is so we put

$$
\int_{F} d \omega=\int_{F_{0}} d \nu d \mu
$$

Then it is clear that

$$
f(1)=\int_{\varepsilon} T_{\omega}(f) d \omega \quad \quad\left(f \in C_{c}^{\infty}(G)\right)
$$

where $T_{\omega}$ is the character of the class $\omega$. Let

$$
g(x)=\int \operatorname{conj}(f(y)) f(y x) d y .
$$

Then $g$ is also in $C_{c}^{\infty}(G)$ and therefore applying the above formula to $g$ we get

$$
g(1)=\int|f(x)|^{2} d x=\int \varepsilon^{N_{\omega}(f) d \omega} \quad\left(f \in C_{c}^{\infty}(G)\right)
$$

where $N_{\omega}(f)$ is defined as in $\S 1$. This gives the Plancherel formula for functions of class $C_{c}^{\infty}(G)$. Since such functions are dense in the Hilbert space $L_{2}(G)$ of all square-integrable functions on $G$, the corresponding formula for functions in $L_{2}(G)$ follows in the usual way by completion.

Now it remains to prove Theorem $4\left(^{9}\right)$. We shall say that a function $\lambda \in F_{-}$ is dominant if $s \lambda \geqq \lambda$ for all $s \in W$ (with respect to the lexicographic ordering defined in the beginning of $\S 5$ ). Let $\mathfrak{F}_{-}^{0}$ be the set of all dominant functions in $\mathfrak{F}_{-}$. Let $\mathfrak{F}^{*}$ be the space of linear functions on $\mathfrak{h}^{*}$. Then $\mathfrak{F}_{+}$and $\mathfrak{F}_{-}$may be regarded as subsets of $\mathfrak{F}^{*}$. Let $\Omega$ be the set of all equivalence classes of finitedimensional simple representations of $K$. A linear function $\mu \in \mathfrak{F}^{*}$ is called a weight of a class $\mathfrak{D} \in \Omega$ if there exists a vector $\psi \neq 0$ in the representation space of any representation $\sigma \in \mathfrak{D}$ such that $\sigma(\exp H) \psi=e^{\mu(H)} \psi\left(H \in \mathfrak{h}_{\mathfrak{t}_{0}}\right)$. We

${ }^{(9)}$ (Added in proof.) A result considerably stronger than Theorem 4 has recently been obtained by F. Bruhat for the classical groups. Since it is possible to give a direct proof of his fundamental lemma (C.R. Acad. Sci. Paris vol. 238 (1954) p. 437) for all connected semisimple Lie groups, his results hold also for the exceptional groups. 
say that $\mu$ is the highest (or lowest) weight of $\mathfrak{D}$ if $\mu+\alpha$ (or $\mu-\alpha$ ) is not a weight of $\mathfrak{D}$ for any $\alpha \in Q$. It is known that every weight lies in $\mathfrak{F}_{-}$and every highest weight in $\mathfrak{F}_{-}^{0}$ (see for example [5, Part I]). Moreover there is a 1-1 correspondence $\Lambda \leftrightarrow \mathfrak{D}_{\Lambda}$ between $\mathfrak{F}_{-}^{0}$ and $\Omega$ such that $\Lambda$ is the highest weight of $\mathfrak{D}_{\Lambda}[5$, Part I $]$. For any $\mu \in F_{-}$let $\delta_{\mu}$ denote the equivalence class of the onedimensional representation $h \rightarrow \xi_{\mu}(h)\left(h \in A_{-}\right)$. Then we denote by $(\Lambda: \mu)$ $\left(\Lambda \in \mathfrak{F}_{-}^{0}\right)$ the number of times $\delta_{\mu}$ occurs in the reduction of $\mathfrak{D}_{\Lambda}$ with respect to $A_{-}$. It is known that $(\Lambda: \Lambda)=1$ and $(\Lambda: \mu)=(\Lambda: s \mu)$ for any $s \in W$ (see Weyl [13]).

Put $\mathfrak{S}=L_{2}(K)$ and define $\mathfrak{S}_{\mathfrak{D}}(\mathfrak{D} \in \Omega)$ to be the set of all elements in $\mathfrak{S}$ which transform according to $\mathfrak{D}$ under the left regular representation of $K$ on $\mathfrak{S}$. As usual we normalise the Haar measures $d u$ and $d h$ on $K$ and $A_{-}$in such a way that $\int_{K} d u=\int_{A_{-}} d h=1$. Let $\lambda$ be any function in $\mathfrak{F}_{-}^{0}$. Put

$$
E_{\lambda}=\int_{A-} \xi_{\lambda}(h) \tau(h) d h
$$

where $\tau$ is the right regular representation of $K$ on $\mathfrak{S}$. Then $E_{\lambda}$ is the orthogonal projection of $\mathfrak{S}$ on $\mathfrak{S}_{\lambda}=E_{\lambda} \mathfrak{S}$. Since $(\lambda: \lambda)=1$, it follows from the Frobenius reciprocity relation (see A. Weil $\left[11\right.$, p. 83]) that $\operatorname{dim}\left(\mathfrak{S}_{\lambda} \cap \mathfrak{S}_{\mathfrak{D}_{\lambda}}\right)=d\left(\mathfrak{D}_{\lambda}\right)$. Therefore apart from a constant factor there is exactly one function $f \neq 0$ in $\mathfrak{S}_{\lambda} \cap \mathfrak{S}_{\mathfrak{D}_{\lambda}}$ such that $f(h u)=\xi_{\lambda}(h) f(u)\left(u \in K, h \in A_{-}\right)$. For any $\mu \in F^{*}$ define a representation $\pi_{\mu}^{\prime}$ of $G$ on $\mathfrak{S}$ by the rule

$$
\pi_{\mu}^{\prime}(x) \phi(u)=\exp \left[-\left\{\left((-1)^{1 / 2} \mu+2 \rho\right)\left(H\left(x^{-1}, u\right)\right)\right\}\right] \phi\left(u_{x^{-1}}\right)
$$

$(u \in K, \phi \in \mathfrak{S}, x \in G)$ in the notation of $\$ 3$. (We recall that $K^{*}=K$ in the present case.) Let $\mathfrak{S}_{\mu, \lambda}$ be the smallest closed subspace of $\mathfrak{S}$ containing $f$ which is invariant under $\pi_{\mu}^{\prime}(G)$. We denote by $\pi_{\mu, \lambda}$ the representation of $G$ induced on $\mathfrak{S}_{\mu, \lambda}$. Since $\tau(h)$ commutes with $\pi_{\mu}^{\prime}(x)\left(h \in A_{-}, x \in G\right)$ it is clear that $\mathfrak{S}_{\mu, \lambda} \subset \mathfrak{S}_{\lambda}$.

LEMMA 15. $\pi_{\nu, \lambda}$ is an irreducible unitary representation of $G$ if $\nu \in F_{+}$. Moreover there exists a set $V_{\lambda}$ in $\mathfrak{F}_{+}$of measure zero such that if $\nu$ lies in the complement of $V_{\lambda}$ in $\mathfrak{F}_{+}, \mathfrak{S}_{\nu, \lambda}=\mathfrak{W}_{\lambda}$.

Let $\nu$ be a function in $\mathfrak{F}_{+}$. Then we have seen that $\pi_{\nu}^{\prime}$, and therefore $\pi_{\nu, \lambda}$, is unitary. We shall now show that $\pi_{\nu, \lambda}$ is irreducible. Suppose $\mathfrak{S}_{\nu, \lambda}=\mathfrak{S}_{1}=\mathfrak{S}_{2}$, where $\mathfrak{S}_{1}, \mathfrak{S}_{2}$ are two mutually orthogonal closed subspaces which are both invariant under $\pi_{\nu, \lambda}(G)$. Then $f=f_{1}+f_{2}\left(f_{i} \in \mathfrak{S}_{i}, i=1,2\right)$ and since $f \neq 0$ we may assume $f_{1} \neq 0$. Then it is clear that $f_{1} \in \mathfrak{S}_{1} \cap \mathfrak{S}_{\mathfrak{D}_{\lambda}}$ and therefore $\operatorname{dim}\left(\mathfrak{S}_{1} \cap \mathfrak{S}_{\mathfrak{D}_{\lambda}}\right) \geqq d\left(\mathfrak{D}_{\lambda}\right)$. But we have seen above that $\operatorname{dim}\left(\mathfrak{S}_{\lambda} \cap \mathfrak{S}_{\mathfrak{D}_{\lambda}}\right)$ $=d\left(\mathfrak{D}_{\lambda}\right)$. Therefore $\mathfrak{S}_{1} \cap \mathfrak{S}_{\mathfrak{D}_{\lambda}}=\mathfrak{S}_{\lambda} \cap \mathfrak{S}_{\mathfrak{D}_{\lambda}}$ and hence $f \in \mathfrak{S}_{1}$. But then it follows from the definition of $\mathfrak{S}_{\nu, \lambda}$ that $\mathfrak{S}_{\nu, \lambda} \subset \mathfrak{W}_{1}$. Therefore $\mathfrak{S}_{\nu, \lambda}$ is irreducible.

In order to prove the second part we need some lemmas. Put $\mathfrak{S}_{0}$ 
$=\sum \mathfrak{D \in \Omega} \mathfrak{S}_{\mathfrak{D}}$. Then every element in $\mathfrak{S}_{0}$ is well-behaved (see Theorem 4 and Lemma 30 of [6]) under $\pi_{\mu}^{\prime}\left(\mu \in \mathfrak{F}^{*}\right)$. Let $\mathfrak{B}$ denote the universal enveloping algebra of $\mathfrak{g}$. We shall also denote by $\pi_{\mu}^{\prime}$ the representation of $\mathfrak{B}$ induced on $\mathfrak{S}_{0}$ (see [6]). Let $\Lambda_{0}$ be a function in $\mathfrak{F}_{-}^{0}$ such that $\mathfrak{S}_{\lambda} \cap \mathfrak{S}_{\mathfrak{D}_{\Lambda_{0}}} \neq\{0\}$. We denote by $(\phi, \psi)$ the scalar product of $\phi, \psi$ in $\mathfrak{S}$.

Lemma 16. Let $\phi_{1}, \cdots, \phi_{m}$ be a base for $\mathfrak{S}_{\lambda} \cap \mathfrak{S}_{\mathbb{D}_{\Lambda_{0}}}$. Suppose $F_{i}, 1 \leqq i \leqq m$, are polynomial functions on $\mathfrak{F}^{*}$ such that

$$
\sum_{i=1}^{m} F_{i}(\mu)\left(\phi_{i}, \pi_{\mu}^{\prime}(b) f\right)=0
$$

for all $b \in \mathfrak{B}$ and $\mu \in \mathfrak{F}^{*}$. Then $F_{i}=0,1 \leqq i \leqq m$.

Notice that

$$
\left(\phi_{i}, \pi_{\mu}^{\prime}(x) f\right)=\int_{K} \operatorname{conj}\left(\phi_{i}(u)\right) \exp \left\{-\left[(-1)^{1 / 2} \mu+2 \rho\right]\left(H\left(x^{-1}, u\right)\right)_{j} f\left(u_{x^{-1}}\right) d u .\right.
$$

Let $X_{1}, \cdots, X_{n}$ be a base for $\mathrm{g}_{0}$ over $R$. Put $X_{t}=t_{1} X_{1}+\cdots+t_{n} X_{n}\left(t_{j} \in R\right)$ and $|t|=\max _{j}\left|t_{j}\right|$. Let $p$ denote any ordered set $\left(p_{1}, \cdots, p_{n}\right)$ of $n$ nonnegative integers. Put $t^{p}=t_{1}^{p_{1}} t_{2}^{p_{2}} \cdots t_{n}^{p_{n}}, p !=p_{1} ! p_{2} ! \cdots p_{n} !$, and

$$
X(p)=\frac{1}{s !} \sum X_{k_{i_{1}}} X_{k_{i_{2}}} \cdots X_{k_{i_{s}}} \in \mathfrak{B}
$$

where $s=p_{1}+p_{2}+\cdots+p_{n},\left(k_{1}, \cdots, k_{s}\right)$ is a sequence of indices in which $j$ occurs exactly $p_{j}$ times $(1 \leqq j \leqq n)$ and the sum is over all permutations $\left(i_{i}, \cdots, i_{s}\right)$ of $(1,2, \cdots, s)$. Then $X(p)$ taken together for all $p$ form a base for $\mathfrak{B}$ (see [4]) and

$$
\left(\phi_{i}, \pi_{\mu}^{\prime}\left(\exp X_{t}\right) f\right)=\sum_{p}\left(\phi_{i}, \pi_{\mu}^{\prime}(X(p)) f\right) \frac{t^{p}}{p !}
$$

provided $|t|$ is sufficiently small (see [6, Theorem 2]). Since $\phi_{i}$ and $f$ are analytic functions on $K$, it follows from the arguments given in the beginning of $\S 4$ of [7] that for each $p$ there exists a polynomial function $\zeta_{i, p}$ on $\mathfrak{F}^{*}$ such that

$$
\left(\phi_{i}, \pi_{\mu}^{\prime}\left(\exp X_{t}\right) f\right)=\sum_{p} \zeta_{i, p}(\mu) \frac{t^{p}}{p !}
$$

provided $|t|$ is sufficiently small. Therefore by comparing coefficients

$$
\left(\phi_{i}, \pi_{\mu}^{\prime}(X(p)) f\right)=\zeta_{i, p}(\mu) .
$$

Since $X(p)$ form a base for $\mathfrak{B}$, it follows that for each fixed $b \in \mathfrak{B}$ the mapping

$$
\mu \rightarrow\left(\phi_{i}, \pi_{\mu}^{\prime}(b) f\right)
$$

is a polynomial function on $\mathfrak{F}^{*}$. 
Now let $\Lambda$ be any function in $\mathfrak{F}_{-}^{0}$. Then $\Lambda+\lambda \in \mathfrak{F}_{-}^{0}$. Let $\mathfrak{D}_{\Lambda}^{*}$ denote the class in $\Omega$ which is contragredient to $\mathfrak{D}_{\Lambda}$. Then $-\Lambda$ is the lowest weight of $\mathfrak{D}_{\Lambda}^{*}$. Choose two representations $\sigma_{1}, \sigma_{2}$ in $\mathfrak{D}_{\Lambda+\lambda}$ and $\mathfrak{D}_{\Lambda}^{*}$ respectively. We denote the corresponding representations of $\mathfrak{f}_{0}$ (and therefore of $\mathfrak{t}$ ) also by the same symbols. Let $U_{1}, U_{2}$ be the representation space of $\sigma_{1}, \sigma_{2}$ respectively. Define a representation $\pi$ of $\mathfrak{g}$ on $U_{1} \times U_{2}$ by the rule $\left({ }^{10}\right)$

$$
\pi\left(\gamma_{+}(X)+\gamma_{-}(Y)\right)=\sigma_{1}(X)+\sigma_{2}(Y)
$$$$
(X, Y \in \mathfrak{t}) .
$$

Then it is clear that $\pi$ is irreducible. Let $G^{\prime}$ be the simply-connected covering group of $G, Z^{\prime}$ the kernel of the natural homomorphism of $G^{\prime}$ on $G$ and $K^{\prime}$ the complete inverse image of $K$ in $G^{\prime}$. Then $K^{\prime}$ is connected and $Z^{\prime} \subset K^{\prime}$ (see Mostow [9]). Now $\pi$ defines a representation of $G^{\prime}$ which we shall also denote by $\pi$. Note that $X=\gamma_{+}(X)+\gamma_{-}(X)(X \in \mathfrak{f})$. Hence

$$
\pi(X)=\sigma_{1}(X)+\sigma_{2}(X)
$$

From this it follows that $\pi\left(u^{\prime}\right)=\sigma_{1}(u) \times \sigma_{2}(u)\left(u^{\prime} \in K^{\prime}\right)$ where $u$ is the image of $u^{\prime}$ in $K$. In particular if $u^{\prime} \in Z^{\prime}, u=1$ and therefore $Z^{\prime}$ is contained in the kernel of $\pi$. Hence $\pi$ may also be regarded as a representation of $G$.

Let $\chi\left(\Lambda^{\prime} ; u\right)(u \in K)$ denote the character of the class $\mathfrak{D}_{\Lambda^{\prime}}\left(\Lambda^{\prime} \in \mathfrak{F}_{-}^{0}\right)$. Then it is evident that

$$
\operatorname{sp} \pi(u)=\chi(\Lambda+\lambda ; u) \operatorname{conj} \chi(\Lambda ; u)
$$$$
(u \in K) \text {. }
$$

For any $\mathfrak{D} \in \Omega$ let $(\pi: \mathfrak{D})$ denote the number of times $\mathfrak{D}$ occurs in the reduction of $\pi(K)$. Then it follows from the Schur orthogonality relations for the characters that

$$
\begin{aligned}
\left(\pi: \mathfrak{D}_{\Lambda_{0}}\right) & =\int_{K} \operatorname{sp} \pi(u) \operatorname{conj} \chi\left(\Lambda_{0} ; u\right) d u \\
& =\int_{K} \chi(\Lambda+\lambda ; u) \operatorname{conj}\left\{\chi\left(\Lambda_{0} ; u\right) \chi(\Lambda ; u)\right\} d u .
\end{aligned}
$$

Now put

$$
\Delta(H)=\prod_{\alpha \in Q}\left(e^{\alpha(H) / 2}-e^{-\alpha(H) / 2}\right) \quad\left(H \in \mathfrak{h}_{\mathfrak{t}_{0}}\right)
$$

and

$$
\Delta(\exp H)=|\Delta(H)| \quad\left(H \in \mathfrak{h}_{\mathfrak{t}_{0}}\right) .
$$

Then we know from the theory of compact Lie groups (see Weyl [13]) that

$$
\left(\pi: \mathfrak{D}_{\Lambda_{0}}\right)=\frac{1}{w} \int_{A_{-}} \chi(\Lambda+\lambda ; h) \operatorname{conj}\left\{\chi(\Lambda ; h) \chi\left(\Lambda_{0} ; h\right)\right\} \Delta^{2}(h) d h
$$

(10) The operations $X$ and + have the same meaning as in [6]. 
where

$$
w=\int_{A-} \Delta^{2}(h) d h=\text { order of } W .
$$

But it is well known (see Weyl [13]) that

$$
\chi\left(\Lambda^{\prime} ; \exp H\right)=\{\Delta(H)\}^{-1} \sum_{s \in W} \epsilon(s) \exp \left\{s\left(\Lambda^{\prime}+\rho\right)(H)\right\} \quad\left(H \in \mathfrak{h}_{\mathfrak{t}_{0}}\right)
$$

for any $\Lambda^{\prime} \in \mathfrak{F}_{-}^{0}$. Here $\epsilon(s)= \pm 1$ and is determined by the rule

$$
\Delta(s H)=\epsilon(s) \Delta(H) \quad\left(s \in W, H \in \mathfrak{h}_{\mathfrak{t}_{0}}\right) .
$$

Therefore

$$
\begin{aligned}
\chi(\Lambda+ & \lambda ; \exp H) \operatorname{conj}\left\{\chi(\Lambda ; \exp H) \chi\left(\Lambda_{0} ; \exp H\right)\right\}\{\Delta(H)\}^{2} \\
= & {\left[\sum_{s, s^{\prime} \in W} \epsilon(s) \epsilon\left(s^{\prime}\right) \exp \left\{s(\Lambda+\lambda+\rho)(H)-s^{\prime}(\Lambda+\rho)(H)\right\}\right] } \\
& \cdot \sum_{j=0}^{r}\left(\Lambda_{0}: \Lambda_{j}\right) \exp \left\{-\Lambda_{j}(H)\right\} \quad\left(H \in \mathfrak{h}_{\mathfrak{t}_{0}}\right)
\end{aligned}
$$

where $\Lambda_{j}, 0 \leqq j \leqq r$, are all the distinct weights of $\mathfrak{D}_{\Lambda_{0}}$.

Now for any function $\mu \in \mathfrak{F}^{*}$ put $\mu\left(H_{t}\right)=t_{1} \mu\left(H_{1}\right)+\cdots+t_{l} \mu\left(H_{l}\right)$ where $t_{1}, \cdots, t_{l}$ are independent indeterminates. Consider the polynomial $S(\mu, t)$ in $(t)$ given by

$$
S(\mu, t)=\prod_{j=0}^{r} \prod_{s \in W, s \neq 1}\left\{(\mu+\lambda+\rho)\left(H_{t}\right)-s(\mu+\rho)\left(H_{t}\right)-\Lambda_{j}\left(H_{t}\right)\right\} .
$$

Then $S(\mu, t)=0$ if and only if

$$
s(\mu+\rho)-(\mu+\rho)=\lambda-\Lambda_{i}
$$

for some $s \neq 1$ in $W$ and some $j$. Since it is obviously possible to choose $\mu$ in such a way that none of these conditions is fulfilled, it follows that $S(\mu, t)$ is not identically zero in $\mu$.

Now suppose the assertion of the lemma is false. Then we may suppose that $F_{1} \neq 0$. Let $F_{1}^{\prime}$ denote the polynomial function on $\mathfrak{F}^{*}$ such that $F_{1}^{\prime}(\mu)$ $=F_{1}\left(\mu^{\prime}\right)$ where $2 \mu+\lambda=-\left[(-1)^{1 / 2} \mu^{\prime}+2 \rho\right]$. Since $F_{1} \neq 0$, it is evident that $F_{1}^{\prime} \neq 0$. Hence $F_{1}^{\prime}(\mu) S(\mu, t)$ is not identically zero in $\mu$. Then the argument of Lemma 32 of [5] is applicable and we can choose $\Lambda \in \mathfrak{F}_{-}^{0}$ such that $F_{1}^{\prime}(\Lambda) S(\Lambda, t) \neq 0$. Then

$$
s(\Lambda+\lambda+\rho)-s^{\prime}(\Lambda+\rho) \neq \Lambda_{i} \quad\left(s, s^{\prime} \in W\right)
$$

for any $j$ unless $s=s^{\prime}$, in which case

$$
s(\Lambda+\lambda+\rho)-s(\Lambda+\rho)=s \lambda .
$$


Hence it follows from the orthogonality of the characters of $A_{-}$that

$$
\left.\left(\pi: \mathfrak{D}_{\Lambda_{0}}\right)\right)=\left(\Lambda_{0}: \lambda\right) \text {. }
$$

Let $U$ be the representation space of $\pi$. We may regard $U$ as a finitedimensional Hilbert space and assume that $\pi(u)$ is unitary for $u \in K$. Put $\pi^{\prime}(x)=\left(\pi\left(x^{-1}\right)\right)^{*}$ where the star denotes adjoint. Then $\pi^{\prime}$ is also an irreducible representation of $G$ on $U$ and $\pi^{\prime}(u)=\pi(u)(u \in K)$. Since $\pi\left(\gamma_{+}(X)+\gamma_{-}(Y)\right)$ $=\sigma_{1}(X)+\sigma_{2}(Y),(X, Y \in \mathfrak{f})$ it is clear that the weights $\left.{ }^{11}\right)$ of $\pi$ (with respect to $\mathfrak{h})$ are exactly the functions $\left(\mu_{1}, \mu_{2}\right)$ where $\mu_{1}$ and $\mu_{2}$ run independently through all weights of $\sigma_{1}$ and $\sigma_{2}$ respectively. Moreover $\left(\mu_{1}, \mu_{2}\right)$ is the highest weight of $\pi$ if and only if $\left(\mu_{1}+\alpha, \mu_{2}\right)$ and $\left(\mu_{1}, \mu_{2}-\alpha\right)$ are not weights of $\pi$ for any $\alpha \in Q$. Hence $\mu_{1}$ must be the highest weight of $\sigma_{1}$ and $\mu_{2}$ the lowest weight of $\sigma_{2}$. Therefore $\mu_{1}=\Lambda+\lambda, \mu_{2}=-\Lambda$. Hence the highest weight of $\pi$ is $(\Lambda+\lambda$, $-\Lambda$ ) and so it coincides with $2 \Lambda+\lambda$ on $\mathfrak{h}_{\mathfrak{p}_{0}}$ and $\lambda$ on $\mathfrak{h}_{\mathfrak{t}_{0}}$. Let $\psi_{0} \neq 0$ be a vector in $U$ belonging to the highest weight. Then $\pi(n) \psi_{0}=\psi_{0}, \pi(h) \psi_{0}=\xi_{\lambda}(h) \psi_{0}$, and $\pi(\exp H) \psi_{0}=\exp \{(2 \Lambda+\lambda)(H)\} \psi_{0}\left(n \in N, h \in A_{-}, H \in \mathfrak{h}_{\mathfrak{p}_{0}}\right)$. Let $\mu$ be the function in $\mathfrak{F}^{*}$ such that

$$
(-1)^{1 / 2} \mu(H)+2 \rho(H)=-\operatorname{conj}(2 \Lambda(H)+\lambda(H)) \quad\left(H \in \mathfrak{h}_{p_{0}}\right) .
$$

For any $\phi \in U$ put

$$
F_{\phi}(u)=\left(\pi(u) \psi_{0}, \phi\right)
$$

where the bracket denotes scalar product in $U$. Then

$$
F_{\pi^{\prime}(x) \phi}(u)=\left(\pi(u) \psi_{0}, \pi^{\prime}(x) \phi\right)=\left(\pi\left(x^{-1} u\right) \psi_{0}, \phi\right)=\pi_{\mu}^{\prime}(x) F_{\phi}(u) .
$$

Moreover if $F_{\phi}=0,\left(\pi(u) \psi_{0}, \phi\right)=0$ for all $u \in K$. But then if $x=u h n(u \in K$, $\left.h \in A_{+}, n \in N\right)$,

$$
\left(\pi(x) \psi_{0}, \phi\right)=\left(\pi(u) \psi_{0}, \phi\right) \exp \left(-\left[(-1)^{1 / 2} \mu+2 \rho\right](\log h)\right)=0 .
$$

Since $\pi$ is an irreducible representation, $U$ is spanned by the transforms $\pi(x) \psi_{0}$ of $\psi_{0}$ and therefore $\phi=0$. Finally

$$
F_{\phi}(u h)=\left(\pi(u h) \psi_{0}, \phi\right)=\operatorname{conj}\left(\xi_{\lambda}(h)\right) F_{\phi}(u) \quad\left(h \in A_{-}\right) .
$$

Therefore $\phi \rightarrow F_{\phi}$ is a 1-1 linear mapping of $U$ into $\mathfrak{S}_{\lambda}$ and $F_{\pi^{\prime}(x) \phi}=\pi_{\mu}^{\prime}(x) F_{\phi}$. Now it is obvious that $\mathfrak{D}_{\Lambda+\lambda}$ occurs in the reduction of $\mathfrak{D}_{\Lambda} \times \mathfrak{D}_{\lambda}$. Therefore $\mathfrak{D}_{\lambda}$ also occurs in the reduction of $\mathfrak{D}_{\Lambda+\lambda} \times \mathfrak{D}_{\Lambda}^{*}$. Since $\pi^{\prime}(u)=\pi(u)(u \in K)$, it follows that there exists a vector $\phi_{0} \neq 0$ in $U$ which transforms according to $\mathfrak{D}_{\lambda}$ under $\pi^{\prime}(K)$ and which is such that $\pi^{\prime}(h) \psi_{0}=\xi_{\lambda}(h) \phi_{0}\left(h \in A_{-}\right)$. Then $F_{\phi_{0}}^{\prime}$ is a nonzero function in $\mathfrak{S}_{\lambda} \cap \mathfrak{S}_{\mathfrak{D}_{\lambda}}$ and

$$
\pi_{\mu}^{\prime}(h) F_{\phi_{0}}=\xi_{\lambda}(h) F_{\phi_{0}}
$$

(11) The weights of $\pi$ are defined as usual (see [5]). They are ordered by the lexicographic ordering introduced by the base $\left(H_{1}, \cdots, H_{l},(-1)^{1 / 2} \Gamma\left(H_{1}\right), \cdots,(-1)^{1 / 2} \Gamma\left(H_{l}\right)\right)$ for $\mathfrak{h}_{p}$ $+(-1)^{1 / 2} \mathfrak{h}_{t_{0}}$ over $R$. 
Since apart from a constant factor, $f$ is the only function in $\mathfrak{S}_{\lambda} \cap \mathfrak{S C D}_{\lambda}$ satisfying this condition $F_{\phi_{0}}=c f$ where $c \in C$ and $c \neq 0$. Then it is evident that the representation $\pi_{\mu, \lambda}$ on $\mathfrak{S}_{\mu, \lambda}$ is equivalent to $\pi^{\prime}$ under the mapping $\phi \rightarrow F_{\phi}$ $(\phi \in U)$. Therefore

$$
\operatorname{dim}\left(\mathfrak{S}_{\mu, \lambda} \cap \mathfrak{S D D}_{\Lambda_{0}}\right)=d\left(\mathfrak{D}_{\Lambda_{0}}\right)\left(\pi: \mathfrak{D}_{\Lambda_{0}}\right)=d\left(\mathfrak{D}_{\Lambda_{0}}\right)\left(\Lambda_{0}: \lambda\right)
$$

since $\pi^{\prime}$ coincides with $\pi$ on $K$. But we know from the Frobenius reciprocity relation (A. Weil $[11$, p. 83]) that

$$
m=\operatorname{dim}\left(\mathfrak{S}_{\lambda} \cap \mathfrak{S}_{\mathfrak{D}_{\Delta_{0}}}\right)=d\left(\mathfrak{D}_{\Lambda_{0}}\right)\left(\Lambda_{0}: \lambda\right) .
$$

Therefore

$$
\mathfrak{S}_{\mu, \lambda} \cap \mathfrak{S}_{\mathbb{D}_{\Lambda_{0}}}=\mathfrak{S}_{\lambda} \cap \mathfrak{S}_{\mathbb{D}_{\Lambda_{0}}}
$$

Since $\pi^{\prime}$ is irreducible and finite-dimensional the same holds for $\pi_{\mu, \lambda}$. Therefore we can choose $b \in \mathfrak{B}$ such that $\sum_{i=1}^{m} F_{i}(\mu) \phi_{i}=\pi_{\mu}^{\prime}(b) f$. Then

$$
\left(\pi_{\mu}^{\prime}(b) f, \pi_{\mu}^{\prime}(b) f\right)=\sum_{i=1}^{m} F_{i}(\mu)\left(\phi_{i}, \pi_{\mu}^{\prime}(b) f\right)=0
$$

and therefore $\pi_{\mu}^{\prime}(b) f=0$. Since $\phi_{i}, 1 \leqq i \leqq m$, are linearly independent over $C$, $F_{1}(\mu)=F_{1}^{\prime}(\Lambda)=0$. But this contradicts our choice of $\Lambda$ and so Lemma 16 is proved.

Now choose the base $\left(\phi_{1}, \cdots, \phi_{m}\right)$ so that it is orthonormal. For any $b \in \mathfrak{B}$ let $\eta_{i, b}$ denote the polynomial function $\mu \rightarrow\left(\phi_{i}, \pi_{\mu}^{\prime}(b) f\right)$ on $\mathfrak{F}^{*}$. Let $J_{0}$ be the ring of all polynomial functions on $\mathfrak{F}^{*}$ and let $J$ be the quotient field of $J_{0}$. Select elements $b_{1}, \cdots, b_{q}$ in $\mathfrak{B}$ such that the matrix $B=\left(\eta_{i, b_{j}}\right)_{1 \leqq i \leqq m, 1 \leqq j \leqq q}$ (with coefficients in $J_{0}$ ) has the maximum possible rank (over $J$ ). We claim this rank is $m$. For otherwise we can find $F_{i} \in J_{0}, 1 \leqq i \leqq m$, not all zero such that

$$
\sum_{i=1}^{m} F_{i} \eta_{i, b j}=0, \quad 1 \leqq j \leqq q .
$$

But in view of the above lemma we can choose $b \in \mathfrak{B}$ such that

$$
\sum_{i=1}^{m} F_{i} \eta_{i, b} \neq 0 \text {. }
$$

Hence if we put $b_{q+1}=b$, the matrix $\left(\eta_{i, b_{j}}\right)_{1 \leqq i \leqq m, 1 \leqq j \leqq q+1}$ has a larger rank than $B$, which contradicts the definition of $B$. Hence $B$ has rank $m$. Therefore $q \geqq m$ and we may assume without loss of generality that $F$ $=\operatorname{det}\left(\eta_{i, b_{j}}\right)_{1 \leqq i, j \leqq m} \neq 0$. Now put

$$
\phi_{j}(\nu)=\sum_{i=1}^{m}\left(\phi_{i}, \pi_{\nu}^{\prime}\left(b_{j}\right) f\right) \phi_{i}=\sum_{i=1}^{m} \eta_{i, b_{j}}(\nu) \phi_{i} \quad\left(\nu \in \mathfrak{F}_{+}\right) .
$$


Since $F \neq 0$, the set $V\left(\Lambda_{0}, \lambda\right)$ of all $\nu \in F_{+}$such that $F(\nu)=0$ is clearly of measure zero. So if $\nu \notin V\left(\Lambda_{0}, \lambda\right), F(\nu) \neq 0$ and therefore $\phi_{j}(\nu), 1 \leqq j \leqq m$, span $\mathfrak{S}_{\lambda}$ $\cap \mathfrak{S}_{\mathcal{D}_{\Lambda_{0}}}$. It is obvious that $\phi_{j}(\nu)$ is the orthogonal component of $\pi_{\nu}^{\prime}\left(b_{j}\right) f$ in $\mathfrak{S O D}_{\mathfrak{D}_{\Lambda_{0}}}$ and therefore it lies in $\mathfrak{S E}_{\nu, \lambda} \cap \mathfrak{S}_{\mathfrak{D}_{\Lambda_{0}}}$. Hence if $\nu \notin V\left(\Lambda_{0}, \lambda\right), \mathfrak{S}_{\lambda} \cap \mathfrak{S}_{\mathcal{D}_{\Lambda_{0}}}$ $=\mathfrak{S}_{\nu, \lambda} \cap \mathfrak{S}_{\mathfrak{D}_{\Lambda_{0}}}$.

Now put $V_{\lambda}=\bigcup_{\Lambda_{0} \in \mathfrak{F}_{-}^{0}} V\left(\Lambda_{0}, \lambda\right)$ where $V\left(\Lambda_{0}, \lambda\right)$ is defined to be the empty set in case $\mathfrak{S}_{\lambda} \cap \mathfrak{S}_{\mathfrak{D}_{\Lambda_{0}}}=\{0\}$. Then $V_{\lambda}$ is a set of measure zero and if $\nu \notin V_{\lambda}$, $\mathfrak{S}_{\lambda} \cap \mathfrak{S}_{\mathfrak{D}_{\Lambda_{0}}}=\mathfrak{S}_{\nu, \lambda} \cap \mathfrak{S D}_{\mathbb{D}_{0}}$ for all $\Lambda_{0} \in \mathfrak{F}_{-}^{0}$. Hence the orthogonal complement of $\mathfrak{S}_{\nu, \lambda}$ in $\mathfrak{W}_{\lambda}$ is zero and therefore $\mathfrak{S}_{\nu, \lambda}=\mathfrak{S}_{\lambda}$. This completes the proof of Lemma 15.

It is clear from the work $\S 3$ that $S_{\nu, \lambda}$ is the character of the representation of $G$ induced on $\mathfrak{S}_{\lambda}$ under $\pi_{\nu}^{\prime}\left(\nu \in \mathfrak{F}_{+}\right)$. Therefore it follows that $S_{\nu, \lambda}$ is an irreducible character if $\nu \in V_{\lambda}$.

So far we have assumed that $\lambda \in \mathfrak{F}_{-}^{0}$. Now let $\lambda$ be any element in $\mathfrak{F}_{-}$. Choose $s \in W$ such that $\lambda_{1}=s \lambda$ is dominant. Then $S_{\nu, \lambda}=S_{s \nu, \lambda_{1}}$ for any $\nu \in \mathfrak{F}_{+}$. We have seen above that there exists a set $V_{\lambda_{1}}$ in $\mathfrak{F}_{+}$of measure zero such that if $s \nu \notin V_{\lambda_{1}}, S_{\nu, \lambda}=S_{s v, \lambda_{1}}$ is an irreducible character. Put $V_{\lambda}=s^{-1} V_{\lambda_{1}}$. Then $V_{\lambda}$ is also of measure zero and if $\nu \notin V_{\lambda}, S_{\nu, \lambda}$ is irreducible. Therefore Theorem 4 is now established.

\section{REFERENCES}

1. C. Chevalley, Theory of Lie groups, Princeton University Press, 1946.

2. - Amer. J. Math. vol. 63 (1941) pp. 785-793.

3. I. M. Gelfand and M. A. Naimark, Trudi Mat. Inst. Steklova vol. 36 (1950).

4. Harish-Chandra, Ann. of Math. vol. 50 (1949) pp. 900-915.

5. —-, Trans. Amer. Math. Soc. vol. 70 (1951) pp. 28-96.

6. - Representations of a semisimple Lie group on a Banach space. I, Trans. Amer. Math. Soc. vol. 75 (1953) pp. 185-243.

7. - Representation of semisimple Lie groups. II, Trans. Amer. Math. Soc. vol. 76 (1954) pp. 26-65.

8. - Representations of semisimple Lie groups. III, Trans. Amer. Math. Soc. vol. 76 (1954) pp. 234-253.

9. G. D. Mostow, Bull. Amer. Math. Soc. vol. 55 (1949) pp. 969-980.

10. L. Schwartz, Theorie des distributions, Paris, Hermann, 1950. 1940.

11. A. Weil, L'Intégration dans les groupes topologiques et ses applications, Paris, Hermann.

12. - C. R. Acad. Sci. Paris vol. 200 (1935) p. 518.

13. H. Weyl, The structure and representation of continuous groups, Princeton, The Institute for Advanced Study, 1935.

Tata Institute of Fundamental Research, BOMBAY, INDIA.

Columbia University, NEW YORK, N. Y. 